\title{
Textures shape the attentional focus: Evidence from exogenous and endogenous cueing
}

\author{
Tobias Feldmann-Wüstefeld • Anna Schubö
}

Published online: 14 August 2013

(C) Psychonomic Society, Inc. 2013

\begin{abstract}
The spatial cueing paradigm (Posner Quarterly Journal of Experimental Psychology 32:3-25, 1980) has often been used to investigate the time course of the deployment of visual attention in space. In a series of eight experiments we investigated whether spatial cues would not only enhance processing of stimuli presented at cued locations, but also enhance processing of the entire texture in which the stimuli were presented. Results showed highest accuracy for responses to stimuli presented at cued locations, a replication of the traditional cueing effect (Posner 1980). Additionally, stimuli presented at uncued locations were responded to with higher accuracy when they were presented inside the same texture as the cued location, as compared with stimuli presented outside the texture with the cued location. To investigate this texture advantage for both automatic and voluntary attention deployment, exogenous and endogenous cues were used. The texture advantage was observed for short interstimulus intervals (ISIs) of 50 and $100 \mathrm{~ms}$ for exogenous cues and for a longer ISI of $200 \mathrm{~ms}$ for endogenous cues. These findings indicate that the arrangement of task-irrelevant visual stimuli also can have a large impact on the cueing effect. This suggests that visual spatial attention spreads texture-wise across the visual field. Control experiments revealed that the homogeneity within texture elements contributes most to the effect but that the texture advantage is a function of both orientation contrast at the texture border and homogeneity within texture elements.
\end{abstract}

Keywords Attention · Texture · Visual search · Cueing · Textons

T. Feldmann-Wüstefeld $(\bowtie) \cdot$ A. Schubö

Department of Experimental and Biological Psychology, Germany Philipps-University Marburg, Marburg, Germany

e-mail: feldmann-wuestefeld@uni-marburg.de

\section{Introduction}

Visual selective attention plays an important role in information processing, since it allows filtering relevant from irrelevant information and, thus, ensures efficient analysis of incoming visual information. It is usually assumed that not all parts of the visual field are attended to the same extent but that certain locations receive prioritized processing, as compared with other locations. Visual spatial attention has been conceptualized as a focus that can be adjusted to the requirements of the visual field and facilitate information processing at focused locations (Eimer, 1999; Eriksen \& St. James, 1986; LaBerge, 1983; Müller \& Hübner, 2002; Posner, 1980; Theeuwes, 2005).

\section{Peripheral and central spatial cueing}

Where the focus of attention is located and how it varies over time has been widely studied using the spatial cueing paradigm introduced by Posner (e.g., Cheal \& Lyon, 1991; Jonides, 1981; Müller \& Rabbitt; 1989; Nakayama \& Mackeben, 1989; Posner, 1980; Posner, Snyder, \& Davidson, 1980; Yantis \& Jonides, 1990). In this paradigm, a task-irrelevant central or peripheral cue precedes a task-relevant target stimulus. A centrally presented symbolic cue (e.g., an arrow) indicates the likely position of the subsequent target. Such a central cue is assumed to induce endogenous (i.e., under voluntary control) shifts of attention that are initiated actively by the observer. On the contrary, an exogenous cue presented in the periphery of the visual field is assumed to capture attention due to its intrinsic properties (e.g., its color, form, or abrupt onset). Peripheral cues can induce exogenous (i.e. involuntary) attention re-allocation in a reflexive manner. Valid cues correctly indicate the target position and cause a benefit (accuracy or response time), as compared with a neutral condition (no cue prior to target), whereas invalid cues 
indicate an incorrect target position and cause a cost, as compared with a neutral condition. These performance differences are assumed to mirror attention deployment to the cued locations (for reviews, see Klein, 2004; Ruz \& Lupiáñez, 2002).

Since uninformative cues (uninformative regarding the target location) can be ignored when the cue is endogenous, but not when it is exogenous (Jonides, 1981), it has been argued that exogenous cues affect the first feedforward sweep of information through the brain in an automatic manner (Lamme, 2000; Marzouki, Grainger, \& Theeuwes, 2007). However, since the magnitude of the cueing effect increases also with increasing informativeness of a peripheral cue, it has been argued that an endogenous component may be involved in exogenous cueing as well (Doallo et al., 2004; Müller \& Rabbitt, 1989; Wright \& Richard, 2000).

Experiments varying the time interval between cue and subsequent (target) stimulus presentation have shown that endogenous and exogenous cues yield attention shifts of different time courses (Eimer, 2000; Funes, Lupiáñez, \& Milliken, 2005; Jonides, 1981; Klein, 2004; Müller \& Findlay, 1988). For exogenous cues, interstimulus intervals (ISIs) of $50 \mathrm{~ms}$ were sufficient to produce a cueing effect, whereas for endogenous cues, an ISI of $200 \mathrm{~ms}$ or more is needed in order to observe a cueing effect (Liu, Stevens, \& Carrasco, 2007; Yeshurun, Montagna, \& Carrasco, 2008). The differential time course might be due to endogenous attention shifts being slower or needing additional time to decode the information carried by the symbolic cue (Eimer, 2000).

\section{The distribution of spatial attention over time}

To account for these results and in order to describe the way attention is deployed in the visual field, the metaphor of an attentional "spotlight" was suggested (Eriksen \& St. James, 1986; Posner, 1980; Posner et al., 1980). According to this metaphor, attention is limited in size and moves independently of eye movements (covert attention; Posner, 1980; Wright \& Ward, 2008) through the visual field. Visual information at locations within this spotlight receives prioritized processing. Later studies suggested that attention is not a strictly delimited area like a spotlight but, rather, a gradient that provides the visual field with various degrees of attention (Downing \& Pinker, 1985; Ghirardelli \& Folk, 1996; Kravitz \& Behrmann, 2008; LaBerge, 1983; Mangun \& Hillyard, 1988; Shulman, Wilson, \& Sheehy, 1985) and that the "shape" of this gradient may be flexibly adjusted to the needs of a specific task (Belopolsky, Zwaan, Theeuwes, \& Kramer, 2007; Theeuwes, 2004).
Attention distribution depending on the structure of the visual field

There is evidence that the distribution of visual selective attention is shaped by the structure of the visual field. For example, it has been suggested that attention is deployed rather to entire objects than to specific spatial locations in the visual field (Duncan, 1984; O'Craven, Downing, \& Kanwisher, 1999). In an experiment by Duncan, two superimposed objects were presented. Each object had two independent attributes. One object was a rectangle that was either small or large and had a gap on either the left- or the right-hand side. The other object was a line that was either dotted or stroked and was tilted to either the left or the right. Observers were to judge attributes of these objects; for example, they had to judge where the rectangle had a gap and/or whether the line was dotted. Participants' performance was equally good for judging one or two attributes of the same object but was impaired for judging two attributes of two different objects (i.e., one attribute for each of the two objects). Because objects were placed at the very same location, Duncan concluded that attention is limited to one object at a time, not a location.

Further evidence for object-based attention stems from a cueing experiment by Egly and colleagues (Egly, Driver, \& Rafal, 1994). Stimulus displays consisted of two drawn-out rectangles, arranged in parallel, and the target consisted of a filled square presented at one of the rectangle's ends. Participants had to press a button as soon as they had detected a target. Prior to the target onset, the outline of one of the four ends of the two rectangles showed an abrupt luminance change, serving as a spatial cue. Cue and target appeared at the same location on $75 \%$ of the trials and at different locations on $25 \%$ of the trials. The critical manipulation was on trials with invalid cues: The target could appear at the other end of the cued rectangle, or it could appear at the equidistant end of the uncued rectangle. Egly et al. found the shortest reaction times (RTs) for validly cued targets, indicating spatial cueing. Moreover, targets presented at locations belonging to the cued object yielded shorter RTs than did targets presented at locations belonging to the noncued object, indicating that attention expanded to the entire object on which the cue had been presented (Egly et al., 1994). As a result, stimuli appearing at the cued object were processed more efficiently than stimuli appearing at a location that did not belong to the cued object-evidence for object-based cueing. In this experiment, the visual field was structured by Gestalt principles of colinearity and closure, but similar results were obtained for colinearity alone (Avrahami, 1999; Marino \& Scholl, 2005). Furthermore, the attention distribution in the visual field was also shown to be shaped by the similarity of objects: Attention more likely spreads from one 
object to a group of objects when they are similar enough (Dodd \& Pratt, 2005). As was pointed out by Ben-Shahar and colleagues (Ben-Shahar, Scholl, \& Zucker, 2007), often, full-fledged, arbitrarily defined objects are used in such experiments, such as bars, rectangles, or circles. However, it is not clear what counts as an object when it comes to the deployment of attention. In the present study, we examined how attention is deployed in the visual field when it is structured not by objects but by basic simple features like line orientation, which underlie objects and virtually any everyday visual scene.

Such simple stimuli have been shown to be grouped preattentively and processed as a unit and are called textons (Ben-Shahar et al., 2007; Julesz, 1986; Nothdurft, 1992, 1993; Wolfe, 1994). Textons allow a segregation of the visual field into distinct areas in an effortless, spontaneous way without the requirement of focal attention, a process called texture segregation (Bergen \& Julesz, 1983; Nothdurft, 1992, 1993; Wolfe, 1992). Texture segregation is one of the prime capabilities of the human visual system (Sagi \& Julesz, 1987; Schubö, Schröger, \& Meinecke, 2007) and is considered distinct from guided or parallel search processes (Wolfe, 1992). Furthermore, it has been shown that observers can divide their attention between two locations within the same texture far better than between two locations within different textures (Ben-Shahar et al., 2007). However, it remains unclear how a visual field that is structured by textures shapes the focus of attention and whether textures induce a gradient of attention.

\section{Rationale of the experiments}

In the present series of eight experiments, we were interested in how attention is deployed in textures of simple oriented lines with texton quality. Since textures can be segregated effortlessly and preattentively (Nothdurft, 1992; Wolfe, 1992), we wanted to examine when and how attention comes into play when observers need to attend particular texture locations. Large arrays of 50 vertical and 50 horizontal lines were arranged in two halves to allow texture segregation on the basis of orientation textons (cf. Fig. 1a). Horizontal and vertical elements were arranged next to each other in such a way that each half consisted of homogeneous elements, resulting in two textures per display. To trigger attention shifts, we employed both exogenous and endogenous cues that are known to enhance processing of stimuli subsequently presented at the cued location for some time (Doallo et al., 2004). We examined whether cues caused enhanced processing at cued locations only or whether the cueing effect would spread to the entire texture to which the cued location belonged. A task-irrelevant oblique line, appearing at one of the four locations, served as an exogenous cue
(Experiments 1 and 4). A centrally presented arrow pointing to one of the four positions served as an endogenous cue (Experiments 2 and 3). After a variable time interval, a blue or green line (the task-relevant target) appeared at one of the four locations. The target could be shown at the same location as the cue (valid trials), at a different location than the cue but inside the same texture (invalid-inside trials), or at a different location than the cue and outside the cued texture (invalid-outside trials). In a series of eight experiments, we examined the impact of cue validity (valid vs. invalid-inside vs. invalid-outside) on the search for the target. Informativeness of the cue (the probability that a cue would correctly indicate the subsequent target position) and cue exposure duration were varied to also examine the role of voluntary attentional control, as compared with more spontaneous attention effects.

We hypothesized that cueing should lead to higher accuracy for targets in invalid-inside trials, as compared with invalid-outside trials. This would support the view that attention is not restricted to the cued location but spreads to the entire texture. It would further argue in favor of an attentional gradient between textures. This logic follows that in Egly et al. (1994), but low-level perceptually coherent textures are considered instead of higher-level objects. We further assumed that in line with traditional cueing experiments (Müller \& Rabbitt, 1989; Posner et al., 1980; Yantis \& Jonides, 1990), performance on valid trials should be better than that on invalid trials. This would argue in favor of an attentional gradient within a texture - that is, more attention at the valid location than at the invalid-inside location. In addition to spatial dynamics, by using several ISIs, we can track down the time course of attention deployment from the starting point (cued location) to various locations in the visual field (e.g., locations inside/outside the texture). In additional experiments, the two determinants of texture segregation, orientation contrast at the texture border and homogeneity within texture elements, were systematically varied (cf. Ben-Shahar et al., 2007; Nothdurft, 1992). Thus, the contribution of both texture properties to shaping the attentional focus in textures could be further assessed.

\section{General method}

\section{Participants}

One hundred twenty-eight volunteers participated in eight experiments and received payment or course credit. The experiments were conducted with the understanding and consent of each participant. All participants had normal or corrected-to-normal vision and had normal color vision (test- 
a

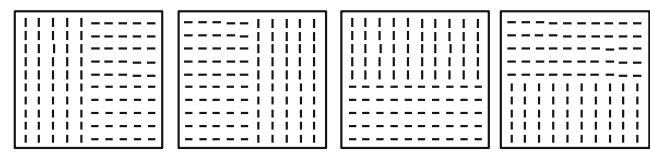

b

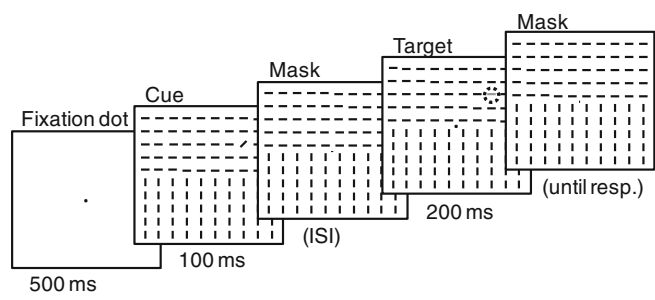

C

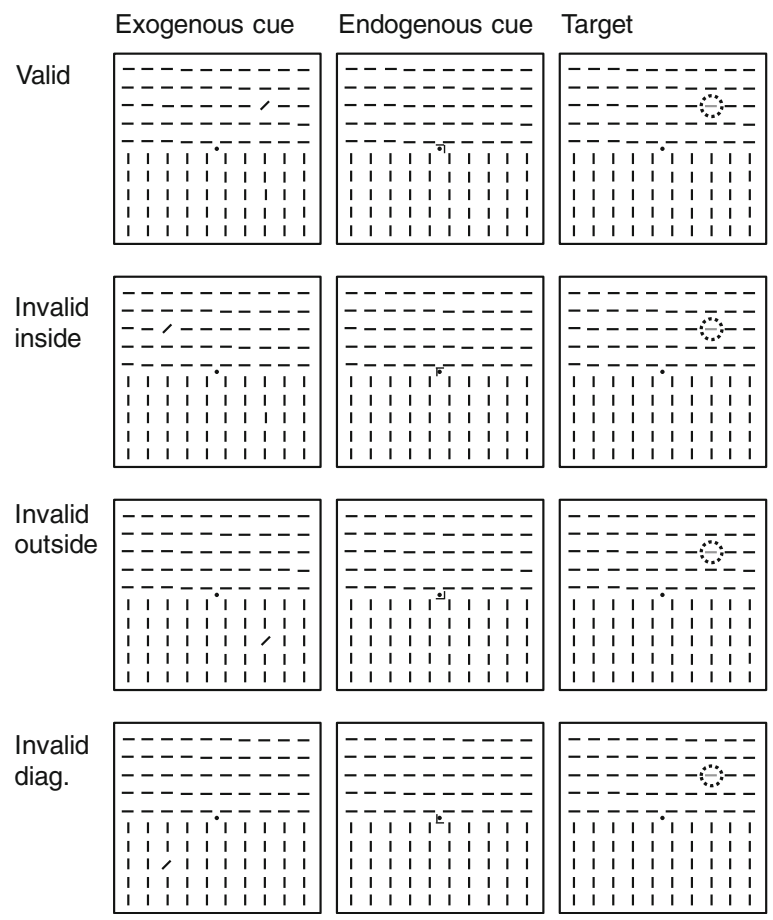

ed via a Rodenstock R12 vision tester, with stimuli no.112 for visual acuity and stimuli no.173 for color vision).

In Experiment 1, 16 participants (5 male) were tested; all were right-handed and between 23 and 27 years of age ( $M=$ $25.4, S D=1.3)$. Experiment 2 comprised 16 participants $(5$ male), all right-handed, 20-29 years of age ( $M=23.2, S D=$ 2.5). Experiment 3 comprised 16 participants (4 male), 12 right-handed, 19-34 years of age $(M=26.1, S D=4.1)$. Experiment 4 comprised 16 participants (5 male), all righthanded, 21-31 years of age $(M=24.0, S D=2.9)$. Experiment 5 comprised 16 participants ( 5 male), all righthanded, $18-28$ years of age $(M=21.4, S D=2.9)$. Experiment 6 comprised 16 participants (4 male), 15 righthanded, $19-35$ years of age $(M=22.9, S D=4.3)$. Experiment 7 comprised 16 participants (5 male), 12 righthanded, $18-28$ years of age $(M=22.6, S D=2.8)$.
Fig. 1 a Illustration of the four texture arrangements used in Experiments 1-6. Horizontal and vertical lines were arranged in such a way that the line stimulus array consisted of either a left and right texture (left panels) or an upper and lower texture (right panels). All four textures were equally likely to appear. b Trial sequence. A trial started with a fixation dot presented for $500 \mathrm{~ms}$, followed by the cue display presented for $100 \mathrm{~ms}$ (Experiment 3: 1,000 ms) and a mask display with a standard texture for a variable interstimulus interval (ISI; cf. Table 1 for specific ISI durations in each experiment). Subsequently, the search display appeared for $200 \mathrm{~ms}$ and was replaced by a mask display that lasted until the participant made a response. $\mathbf{c}$ Illustration of the four validity conditions used in Experiments 1-4. On valid trials, the cue and target appeared at the same location. On invalid-inside trials, the cue and target appeared at different locations, and the target was presented inside the same texture. On invalid-outside trials, the cue and target appeared at different locations, but the target was outside the cued texture. Note that the distance between the cue and target was identical on invalid-inside and invalid-outside trials. On invalid-diagonal trials, the cue and target appeared at different locations, and the target was diagonally shifted relative to the cue. Exogenous cues (Experiments 1, 4-8) were diagonal line elements presented at one of four possible peripheral cue locations (left columns). Endogenous cues (Experiments 2 and 3) were centrally presented L-shaped arrows pointing toward one of these four locations (middle columns). Targets were green or blue line elements (a dotted line encircles the target locations for illustration purposes and was not visible to the participants), oriented in the same way as surrounding line elements

Experiment 8 comprised 16 participants (4 male), all righthanded, $19-33$ years of age $(M=23.3, S D=2.7)$

Stimuli and apparatus

Participants were seated in a comfortable chair in a dimly lit, electrically shielded, and sound-attenuated chamber, with an ergonomic gamepad (Microsoft Sidewinder USB) in their hands. Two buttons on the backside of the gamepad had to be pressed with the left and right index fingers. All stimuli were presented on a 19 -in. computer screen with a $100-\mathrm{Hz}$ refresh rate placed at a distance of $85 \mathrm{~cm}$ from the observer. ${ }^{1} \mathrm{~A}$ light gray served as background for all displays. A trial consisted of two consecutive displays, a cue and a search display. Cue displays consisted of a matrix of $10 \times 10$ stimuli,

\footnotetext{
${ }^{1}$ Due to the fact that both authors changed affiliation during the experimental series, different screens were used in Experiments 1-4 and 5-8. Instead of a 19-in. CRT screen, a 22-in. LCD (TN panel; $100 \mathrm{~Hz}$ ) screen was used. To maintain the visual angle of all stimuli, the distance to the screen was set to $100 \mathrm{~cm}$. Preliminary tests showed, however, that with these settings, performance was much better than in Experiments 1-4, presumably due to the difference between CRT and LCD screens. Thus, search display duration was decreased and, to further avoid ceiling or floor effects, adjusted for each participant separately in a step function: From a starting point of $100 \mathrm{~ms}$, participants had to perform between $65 \%$ and $85 \%$ correct for two succeeding practice blocks of 32 trials. If performance exceeded this criterion in any single block, search display duration was increased or decreased by $20 \mathrm{~ms}$. The criterion of $65 \%-85 \%$ was chosen on the basis of Experiment 4 (here, mean accuracy across all conditions was $75.9 \%$ $( \pm 1 S D=10.9 \% \approx 65 \% / 85 \%)$, which used exogenous uninformative cues like Experiments 5-8.
} 
50 of which were horizontal and 50 of which were vertical dark gray lines. Horizontal and vertical lines were arranged in two halves, with horizontal and vertical lines separated in either an upper and lower or a left and right half of the field (cf. Fig. 1a). Both types of displays (separation into an upper and lower or a left and right half) were presented with equal probability. Line length was $1.1^{\circ}$, and the matrix had a length and height of $14.8^{\circ}$. A single oblique line element, tilted $45^{\circ}$ clockwise or counterclockwise, served as the exogenous cue in Experiments 1 and 4-8 (Fig. 1c, left column). A centrally presented equal-sided L-shaped arrow pointing to one of the four quadrants was used as an endogenous cue in Experiments 2 and 3 (Fig. 1b, middle column). The cue indicated one of four positions at $7.7^{\circ}$ eccentricity at the center of each of the four imaginary quadrants. Search displays were identical to the cue displays, except that no cue was presented and one of the horizontal or vertical lines was colored either green or blue instead (each $50 \%$ ), serving as the target (Fig. 1c, right column). Targets appeared equally often at the four potential cue positions. This led to four possible validity conditions: (1) On valid trials, cues correctly indicated the target position (e.g., Fig. 1b, upper row); (2) on invalid-inside trials, the cue indicated a position different from the actual target position, horizontally or vertically shifted by $15.4^{\circ}$ but inside the homogeneous texture (e.g., Fig. 1c, second row); (3) on invalidoutside trials, the cue indicated a position different from the actual target position, horizontally or vertically shifted by $15.4^{\circ}$ but outside the cued texture (e.g., Fig. 1c, upper row); (4) on invalid-diagonal trials, the cue indicated a position different from the actual target position, diagonally shifted by $21.8^{\circ}$. After cue offset and after search display offset, a "standard" line array of horizontal and vertical lines was presented without marked cue or target.

\section{Procedure}

A trial started with the presentation of a central fixation dot $(2 \times 2$ pixels) that remained on the screen throughout the entire trial (see Fig. 1b for an exemplary trial sequence). After $500 \mathrm{~ms}$, the cue display was presented. Cue presentation time varied between experiments (see Table 1). Subsequently, a standard texture was shown for a variable ISI (see Table 1) to serve as a mask before the search display appeared for $200 \mathrm{~ms}$ (see note 1) on the screen and was subsequently replaced by the standard texture, again serving as a mask. Participants were to press one of the response buttons (labeled "GREEN" or "BLUE") in order to indicate the color of the target in the search display; response accuracy was emphasized, and there was no time limit for the response. After a response was given, a blank screen (light gray background) was shown for $300 \mathrm{~ms}$ until a fixation cross indicated the beginning of the next trial. Response assignment (left vs. right index finger) was balanced across participants. All four validity conditions (valid, invalidinside, invalid-outside, and invalid-diagonal) were combined with four ISIs, leading to 16 conditions in each experiment (see Table 1). All types of trials were randomly assigned to blocks of 32 trials each (see Table 1 for number of trials). After each block, performance feedback (response accuracy) was given, followed by a short break of at least $10 \mathrm{~s}$.

\section{Data analysis}

Mean accuracy was computed for each participant separately for all 16 conditions, excluding trials with false responses and trials with RTs longer than the participant's mean RT $\pm 2 S D$ s. For RT analyses, trials with erroneous responses or with RTs longer than the subject's mean RT \pm $2 S D$ s were excluded. An ANOVA was calculated with the factors ISI and validity (valid vs. invalid-inside vs. invalid-outside vs. invalid-diagonal) for accuracy and RTs. Greenhouse-Geisser correction was used when appropriate.

\section{Experiment 1}

Experiment 1 examined whether performance for targets presented at uncued locations was better when targets belonged to the same texture as the cue. A single oblique line element tilted $45^{\circ}$ clockwise or counterclockwise served as an exogenous cue. Targets appeared at the cued location on $72.7 \%$ of all trials (valid trials) and at an uncued location on $27.3 \%$ of all trials ( $9.1 \%$ for each invalid condition). The ISI between cue and search display was 50, 100, 150, or $200 \mathrm{~ms}$. We expected valid trials to result in the highest accuracy, in compliance with the traditional cueing effect (Posner, 1980). More important, if cues cause enhanced processing not only at the cued locations but also for entire groups of similar stimuli, invalid-inside trials should result in higher accuracies than should invalid-outside trials.

\section{Results}

Accuracy (cf. Fig. 2a and Table 2)

Valid cues led to most accurate performance $(M=88.1 \%)$, followed by invalid-inside $(M=62.3 \%)$, invalid-outside $(M=60.0 \%)$, and invalid-diagonal $(M=58.0 \%)$ trials, $F(1.7,25.3)=57.37, p<.001, \eta^{2}=.79$. The most accurate performance was reached for an ISI of $50 \mathrm{~ms}(M=69.8 \%)$, followed by ISIs of $100 \mathrm{~ms}(M=68.2 \%), 150 \mathrm{~ms}(M=$ $66.6 \%)$, and $200 \mathrm{~ms}(M=63.8 \%), F(3,45)=4.41, p=.008$, $\eta^{2}=.23$. The interaction of both factors also reached significance, $F(9,135)=2.19, p=.026, \eta^{2}=.13$. Planned contrasts 
Table 1 Overview of experimental settings in Experiments 1-8

Experiment 1 Experiment 2 Experiment 3 Experiment 4 Experiment 5 Experiment 6 Experiment 7 Experiment 8

\begin{tabular}{|c|c|c|c|c|c|c|c|c|}
\hline Cue Type & Exogenous & Endogenous & Endogenous & Exogenous & Exogenous & Exogenous & Exogenous & Exogenous \\
\hline Informativeness & $72.7 \%$ & $72.7 \%$ & $72.7 \%$ & $25 \%$ & $25 \%$ & $25 \%$ & $25 \%$ & $25 \%$ \\
\hline Inter-Stimulus & $50 / 100 /$ & $100 / 200 /$ & $100 / 200 /$ & $50 / 100 /$ & $0 / 50 / 100 /$ & $50 / 100 /$ & $150 / 200$ & $150 / 200$ \\
\hline Interval [ms] & $150 / 200$ & $400 / 600$ & $400 / 600$ & $150 / 200$ & $150 / 200$ & $150 / 200$ & & \\
\hline Cue Duration [ms] & 100 & 100 & 1,000 & 100 & 100 & 100 & 100 & 100 \\
\hline No. of trials (blocks) & $1,408(44)$ & $1,408(44)$ & $1,408(44)$ & $1,024(32)$ & $1,024(32)$ & $1,024(32)$ & $1,024(32)$ & $1,024(32)$ \\
\hline $\begin{array}{l}\text { Texture Homogeneity: } \\
\text { Cue display }\end{array}$ & $0^{\circ}$ & $0^{\circ}$ & $0^{\circ}$ & $0^{\circ}$ & $0^{\circ}$ & $0^{\circ}$ & $0^{\circ}$ & $30^{\circ} / 90^{\circ}$ \\
\hline $\begin{array}{l}\text { Texture Homogeneity: } \\
\text { Search display }\end{array}$ & $0^{\circ}$ & $0^{\circ}$ & $0^{\circ}$ & $0^{\circ}$ & $0^{\circ}$ & $0^{\circ}$ & $30^{\circ} / 90^{\circ}$ & $30^{\circ} / 90^{\circ}$ \\
\hline Border Contrast & $90^{\circ}$ & $90^{\circ}$ & $90^{\circ}$ & $90^{\circ}$ & $90^{\circ}$ & $90^{\circ}$ & $90^{\circ}$ & $30^{\circ} / 90^{\circ}$ \\
\hline $\begin{array}{l}\text { Change of Texture } \\
\text { Arrangement }\end{array}$ & no & no & no & no & yes & no & no & no \\
\hline $\begin{array}{l}\text { Change of Texton } \\
\text { Identity }\end{array}$ & no & no & no & no & no & yes $\left(+90^{\circ}\right)$ & yes (gradual) & no \\
\hline
\end{tabular}

Note. "Cue Type" describes whether cues were exogenous (diagonal line elements presented at one of four possible peripheral cue locations) or endogenous (centrally presented L-shaped arrows pointing toward one of these four locations). "Informativeness" denotes the probability that the cue correctly indicated the subsequent target location. "Interstimulus interval" marks the time between cue offset and target onset. "Texture Homogeneity: Cue display" denotes the homogeneity within texture elements in the cue display- that is, the orientation variation of line elements within a texture $\left(0^{\circ}\right.$ equals no variation). "Texture Homogeneity: Search display" denotes the same variation of homogeneity for the search display. "Border Contrast" describes the orientation contrast at the texture border - that is, the orientation difference of neighboring line elements at the border between textures. "Change of Texture Arrangement" specifies whether the texture arrangement switched from horizontal to vertical (or vice versa) between the cue and search displays or whether the arrangement remained the same. "Change of Texton Identity" specifies whether the identity of all texture elements (the textons) switched from horizontal to vertical (or vice versa) between the cue and search displays or whether all textons remained the same. Bold font indicates that an experimental variation was unique for a particular experiment.

revealed a significant difference in accuracy between invalidinside and invalid-outside trials for an ISI of $50 \mathrm{~ms}\left(M_{\text {inside }}=\right.$ $66.8 \%$ vs. $\left.M_{\text {outside }}=60.1 \%\right), p=.007, \varepsilon=0.99$, but not for longer ISIs (all $p \mathrm{~s}>.124$ ).

\section{Reaction times (cf. Table 2)}

There was no effect of validity, $F(1.1,17.1)=2.84, p=$ $.107, \eta^{2}=.16$. The shortest RTs were found for an ISI of $200 \mathrm{~ms}(315 \mathrm{~ms})$, followed by ISIs of $150 \mathrm{~ms}(335 \mathrm{~ms})$, $100 \mathrm{~ms}(376 \mathrm{~ms})$, and $50 \mathrm{~ms}(415 \mathrm{~ms}), F(3,45)=73.21$, $p<.001, \eta^{2}=.83$. Also, the interaction of both factors reached significance, $F(3.9,58.6)=3.32, p=.017, \eta^{2}=$ .18 , but planned contrasts revealed no significant difference between invalid-inside and invalid-outside trials for any ISI (all $p \mathrm{~s}>.05$ ).

\section{Discussion}

As was expected, exogenous cues led to higher accuracy for targets presented at the cued location (valid trials), as compared with any other position of the visual field (invalid trials). This shows that the traditional cueing effect (Posner, 1980) can also be evoked by odd elements in otherwise homogeneous textures. Improved performance for valid, as compared with invalid, trials was observed for all ISIs used, although the effect was slightly attenuating from the shortest $(50 \mathrm{~ms})$ to the longest $(200 \mathrm{~ms})$ ISI. More interesting, however, is that target accuracy on invalid trials also depended on whether target and cued location were part of the same texture: For an ISI of $50 \mathrm{~ms}$, accuracy on invalid trials was higher when the target was presented in the same texture as the cue, as compared with when the target was presented outside that texture. We call this finding the texture advantage. For longer ISIs, targets did not benefit from being part of the same texture as the cue.

\section{Experiment 2}

Experiment 1 showed that exogenous cues lead to better performance on invalid-inside, as compared with invalidoutside, trials. The aim of Experiment 2 was to examine whether this texture advantage would be observed for endogenous cues as well. Experiment 2 was identical to Experiment 1, except that a centrally presented L-shaped arrow pointing to one of the four target positions served as an endogenous cue and longer ISIs were used that ranged between 100 and $600 \mathrm{~ms}$ (see Table 1). 

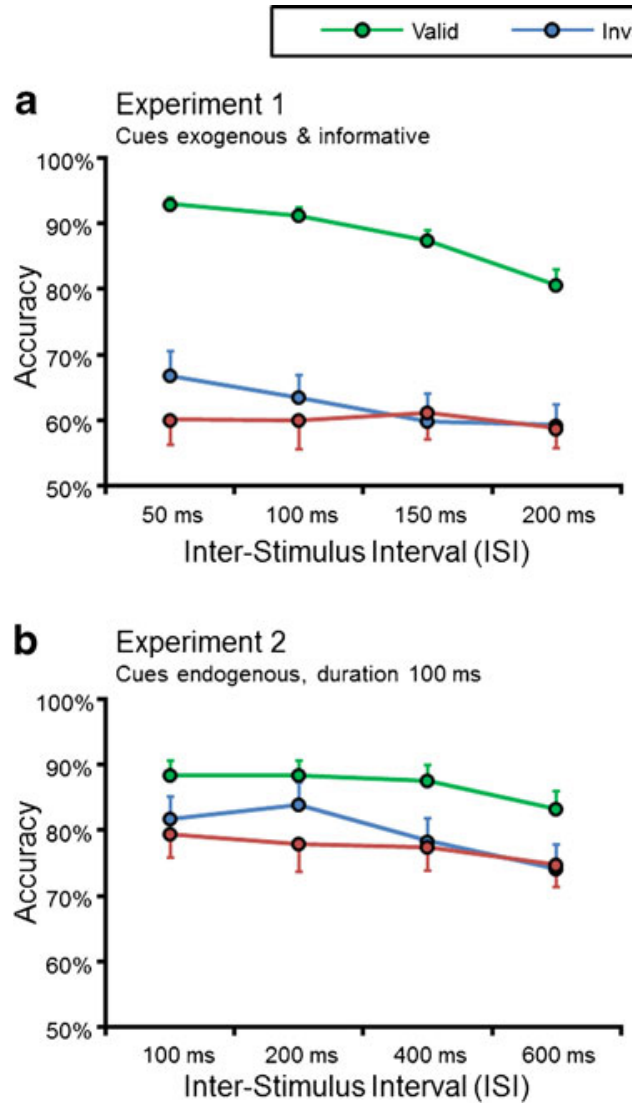

Fig. 2 Mean accuracy in Experiment 1 (upper left panel), Experiment 2 (lower left panel), Experiment 3 (upper right panel), and Experiment 4 (lower right panel). Accuracy is shown as a function of interstimulus

\section{Results}

\section{Accuracy (cf. Fig. $2 b$ and Table 2)}

As was expected, valid cues led to the best performance $(M=$ $86.8 \%)$, followed by invalid-inside $(M=79.5 \%)$, invalidoutside $(M=77.4 \%)$ and invalid-diagonal $(M=75.8 \%)$ trials, $F(2.1,31.0)=7.70, p=.002, \eta^{2}=.34$. Performance was most accurate for an ISI of $100 \mathrm{~ms}(M=82.8 \%)$, followed by ISIs of $200 \mathrm{~ms}(M=81.2 \%), 400 \mathrm{~ms}(M=80.0 \%)$, and $600 \mathrm{~ms}(M=$ $75.6 \%), F(3,45)=11.06, p<.001, \eta^{2}=.42$. The interaction of both factors did not reach significance $(p=.153)$. Planned contrasts, however, revealed a significant difference in accuracy between invalid-inside and invalid-outside trials for an ISI of $200 \mathrm{~ms}\left(M_{\text {inside }}=83.8 \%\right.$ vs. $\left.M_{\text {outside }}=77.9 \%\right), p=.003, \varepsilon=$ 1.12 , but not for ISIs of 100,400 , or $600 \mathrm{~ms}$ (all $p \mathrm{~s}>.214$ ).

\section{Reaction times (cf. Table 2)}

Valid cues led to the shortest RTs (507 ms), followed by invalid-inside (606 ms), invalid-outside $(609 \mathrm{~ms})$, and invalid-diagonal $(613 \mathrm{~ms})$ trials, $F(1.8,26.3)=10.91$, $p=.001, \eta^{2}=.42$. For an ISI of $600 \mathrm{~ms}$, the shortest RT were
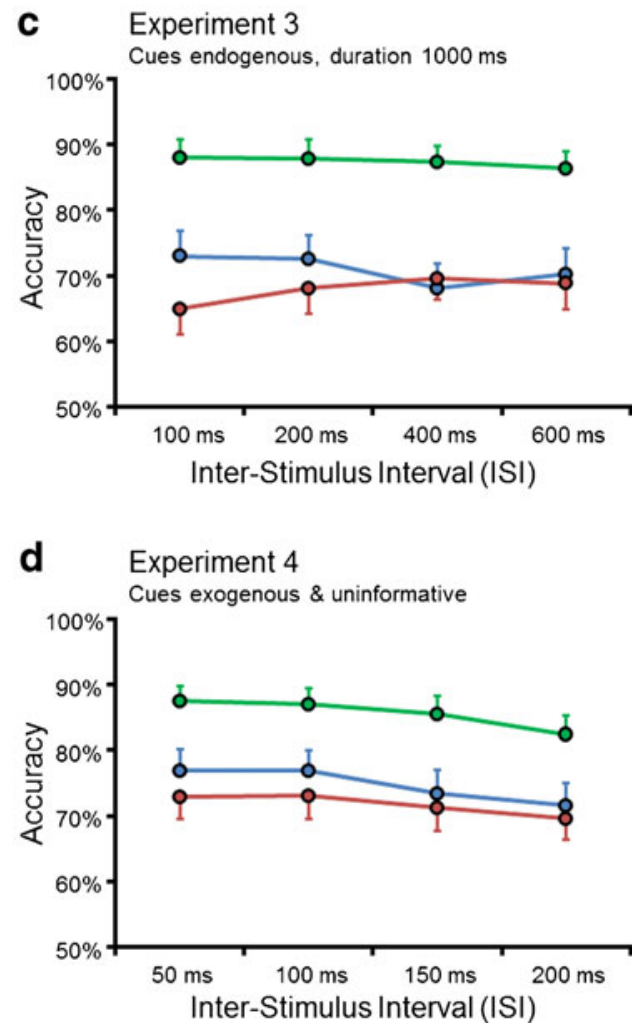

interval and validity (valid, green lines; invalid-inside, blue lines; invalid-outside, red lines; for invalid-diagonal trials, see Table 2). Error bars indicate the standard errors of the means

found (508 ms), followed by ISIs of $400 \mathrm{~ms}$ ( $570 \mathrm{~ms}), 200 \mathrm{~ms}$ (606 ms), and $100 \mathrm{~ms}(651 \mathrm{~ms})$ [main effect of ISI, $F(1.5,22.2)$ $\left.=18.65, p<.001, \eta^{2}=.55\right]$. The interaction of both factors did not reach significance $(p=.376)$; neither did planned contrasts reveal a significant difference between invalid-inside and invalid-outside trials for any ISI (all $p \mathrm{~s}>.05$ ).

\section{Discussion}

Similar to Experiment 1, valid cues led to more accurate performance than did invalid cues in Experiment 2, replicating earlier studies (Klein, 2004; Ruz \& Lupiáñez, 2002). More important, also endogenous cues yield a texture advantage: Accuracy on invalid trials was higher when the target was presented in the same texture as the cued location, as compared with when the target was presented outside that texture. For endogenous cues, texture advantage was observed much later than for exogenous cues - namely, for an ISI of $200 \mathrm{~ms}$. For the shortest ISI of 100 and the two longer ISIs of 400 and $600 \mathrm{~ms}$, no texture advantage was found. Comparing Experiments 1 and 2, it becomes evident that endogenous cues need more time than do exogenous cues to evoke a texture advantage. 
Table 2 Overview of mean accuracy and mean reaction times in Experiments 1-8

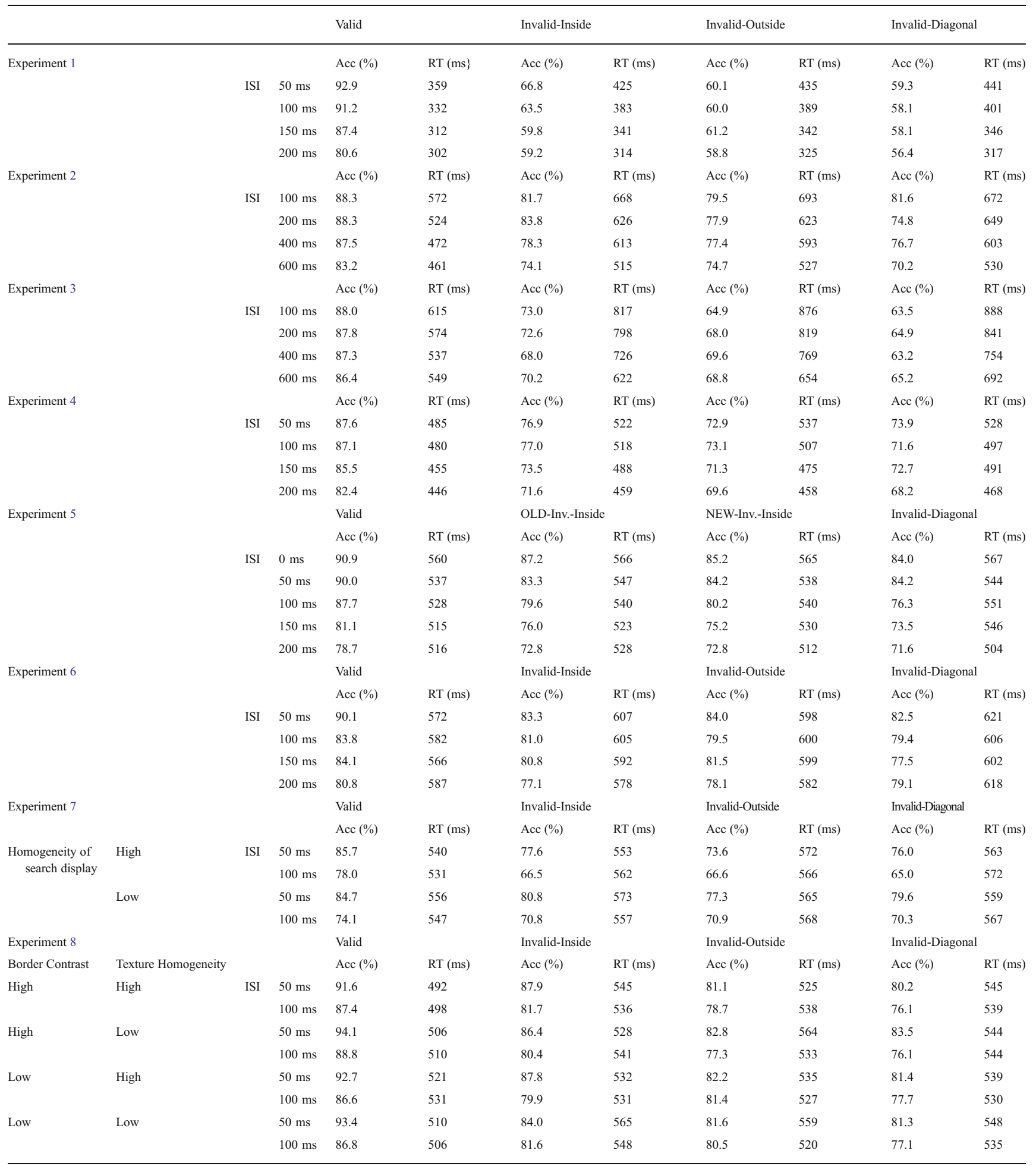

Note. The first column to the left indicates the number of the experiment, and the second column indicates the interstimulus interval (ISI). Columns 3-6 show the mean accuracy (Acc) in percent correct and the mean reaction times (RT) in milliseconds across all participants for valid, invalid-inside, invalid-outside, and invalid-diagonal trials. Note that in Experiment 5, texture border switches from horizontal to vertical (or vice versa), resulting in OLD-invalid-inside and NEW-invalid-inside trials (see the Experiment 5 section for details). In Experiment 7, the additional factor "Homogeneity of search display" was varied while the homogeneity of the cue display remained homogeneous (as in Experiments 1-5). High homogeneity denotes an orientation difference of $30^{\circ}$ within a texture (low homogeneity: $90^{\circ}$ ). In Experiment 8, the two additional factors, "Border Contrast" and "Texture Homogeneity," were varied. High border contrast denotes a $90^{\circ}$ orientation difference between neighboring lines at the border of the texture (low border contrast: $30^{\circ}$ ). High homogeneity denotes an orientation difference of $30^{\circ}$ within a texture (low homogeneity: $90^{\circ}$ ). 


\section{Experiment 3}

Experiment 2 revealed a cueing effect on valid trials already after an ISI of $100 \mathrm{~ms}$. A texture advantage, however, was observed only later, after an ISI of $200 \mathrm{~ms}$. One may therefore wonder how the texture advantage relates to traditional spatial cueing and what time course both effects follow. One may speculate that a cue presentation time of $100 \mathrm{~ms}$ and an ISI of $100 \mathrm{~ms}$ were enough time to induce traditional spatial cueing on valid trials but too short to induce a texture advantage. Alternatively, one may assume that the texture advantage may be a by-product of failed spatial cueing; that is, participants may have had problems in focusing their attention on the cued location but have attended the entire texture. Experiment 3 was designed to investigate whether an increase in cue presentation time to $1,000 \mathrm{~ms}$ would change the cueing effect pattern and, especially, whether it would modulate the texture advantage. With this increase, participants would have enough time to focus their attention on the cued location and would not need to attend the entire element texture. Contrarily, the presence of a texture advantage with a longer preparation interval would speak in favor of a texture effect independent of traditional spatial cueing.

\section{Results}

\section{Accuracy (cf. Fig. 2c and Table 2)}

Again, valid cues led to the highest accuracy $(M=87.4 \%)$, followed by invalid-inside $(M=70.9 \%)$, invalid-outside $(M=$ $67.8 \%)$, and invalid-diagonal $(M=64.2 \%)$ trials, $F(1.3,19.5)=$ $20.05, p<.001, \eta^{2}=.57$. No other effects reached significance (all $p$ s $>$.241). Planned contrasts revealed a significant difference for invalid-inside and invalid-outside trials for an ISI of $100 \mathrm{~ms}\left(M_{\text {inside }}=73.0 \%\right.$ vs. $\left.M_{\text {outside }}=64.9 \%\right), p=.002, \varepsilon=$ 1.20 , and for an ISI of $200 \mathrm{~ms}\left(M_{\text {inside }}=72.6 \%\right.$ vs. $M_{\text {outside }}=$ $68.0 \%), p=.003, \varepsilon=1.11$, but not for an ISI of 400 or $600 \mathrm{~ms}$ (all $p \mathrm{~s}>.05)$.

\section{Reaction times (cf. Table 2)}

Valid cues led to the shortest RTs ( $569 \mathrm{~ms})$, followed by invalidinside (741 ms), invalid-outside (779 ms), and invalid-diagonal $(794 \mathrm{~ms}), F(1.2,18.7)=17.60, p<.001, \eta^{2}=.54$. For an ISI of $600 \mathrm{~ms}$, the shortest RTs were found $(629 \mathrm{~ms})$, followed by ISIs of $400 \mathrm{~ms}$ (696 ms), $200 \mathrm{~ms}$ (758 ms), and $100 \mathrm{~ms}$ (799 ms), $F(1.5,22.3)=28.09, p<.001, \eta^{2}=.65$. There was an interaction of ISI and validity, $F(4.4,66.7)=2.75, p=.031, \eta^{2}=.16$, There was an interaction of ISI and validity, $F(4.4,66.7)=2.75$, $p=.031, \eta^{2}=.16$, and planned contrasts revealed a significant difference between invalid-inside and invalid-outside for an ISI of $100 \mathrm{~ms}\left(M_{\text {inside }}=817 \mathrm{~ms}\right.$ vs. $\left.M_{\text {outside }}=876 \mathrm{~ms}\right), p=.015$, $\varepsilon=0.84$, but for no other ISI (all $p \mathrm{~s}>.081$ ).
Discussion

As in Experiment 2, ISIs of 400 and $600 \mathrm{~ms}$ showed no texture advantage in the present experiment. Interestingly, however, while in Experiment 2 a texture advantage was found for an ISI of $200 \mathrm{~ms}$ but not earlier, Experiment 3 showed that an increase in cue presentation time can cause a texture advantage already for an ISI of $100 \mathrm{~ms}$. In both Experiments 2 and 3, an optimal strategy would have been to focus on the cued location (target probability of $73 \%$ ), while all other positions were equally "unlikely" to be followed by the target (each $9 \%$ ). Even though participants had enough time now to focus their attention accordingly, accuracy was still higher on invalid-inside than on invalid-outside trials. This indicates that the texture had an impact on search performance in addition to the Posnerian cueing effect. In fact, a texture advantage was now observed already after a shorter ISI of $100 \mathrm{~ms}$. The increase in cue presentation time thus did not abolish the texture advantage but, rather, made it appear at an earlier time interval. This strongly speaks in favor of a texture effect independent of traditional spatial cueing.

\section{Experiment 4}

Is it possible that the texture advantage observed in the previous experiments was entirely due to endogenous control mechanisms? In Experiment 1, exogenous cues were used that are considered to cause automatic attention shifts (Klein, 2004; Marzouki et al., 2007). However, it is often argued that exogenous cues may also induce endogenous control to some extent when they are informative (Doallo et al., 2004; Müller \& Rabbitt, 1989; Wright \& Richard, 2000). The aim of Experiment 4 was to test whether a texture advantage is also observed without endogenous control being involved. To do so, the experimental design of Experiment 1 was modified so that the target would appear at each of the four positions with a probability of $25 \%$. Hence, the cue was uninformative with respect to the location of the subsequent target, and participants would not benefit from attending to one position more than to another, since the target could appear at any position with equal probability.

\section{Results}

Accuracy (cf. Fig. 2d and Table 2)

Valid cues led to the best performance $(M=85.6 \%)$, followed by invalid-inside $(M=74.7 \%)$, invalid-outside $(M=71.7 \%)$, and invalid-diagonal $(M=71.6 \%)$ trials, $F(1.3,19.2)=21.37$, $p<.001, \eta^{2}=.59$. Best performance was reached for an ISI of $50 \mathrm{~ms}(M=77.8 \%)$, followed by ISIs of $100 \mathrm{~ms}(M=77.2 \%)$, $150 \mathrm{~ms}(M=75.7 \%)$, and $200 \mathrm{~ms}(M=73.0 \%), F(2.0,30.6)=$ 
20.46, $p<.001, \eta^{2}=.58$. There was no interaction of both factors, $p=.857$.

To directly compare accuracy performance between the crucial conditions invalid-inside and invalid-outside, planned contrasts were calculated for each of the four ISIs separately. Accuracy differed significantly for an ISI of $100 \mathrm{~ms}\left(M_{\text {inside }}=\right.$ $77.0 \%$ vs. $M_{\text {outside }}=73.1 \%$ ), $p=.004, \varepsilon=1.08$, and for an ISI of $50 \mathrm{~ms}\left(M_{\text {inside }}=76.9 \%\right.$ vs. $\left.M_{\text {outside }}=72.9 \%\right), p=.009$, $\varepsilon=0.93$, but not for an ISI of 150 or $200 \mathrm{~ms}$ (all $p \mathrm{~s} \geq .05$ ).

\section{Reaction times (cf. Table 2)}

Valid cues led to the shortest RTs (466 ms), while responses differed only slightly between the other three conditions: invalid-inside (497 ms), invalid-outside (494 ms), and invaliddiagonal $(496 \mathrm{~ms}), F(3,45)=10.07, p<.001, \eta^{2}=.40$. For ISIs of $200 \mathrm{~ms}$, the shortest RT were found (458 ms), followed by ISIs of $150 \mathrm{~ms}(477 \mathrm{~ms}), 100 \mathrm{~ms}(500 \mathrm{~ms})$, and $50 \mathrm{~ms}(518 \mathrm{~ms})$, $F(1.9,28.1)=17.94, p<.001, \eta^{2}=.55$. There was no interaction of ISI and validity $(p=.098)$, and planned contrasts revealed no significant difference of the means between invalid-inside and invalid-outside for any ISI (all $p \mathrm{~s}>.05$ ).

\section{Comparison of Experiments 1 and 4}

To assess possible differences in the texture advantage due to cue informativeness, a post hoc ANOVA with the withinsubjects factor validity and the between-subjects factor informativeness (Experiment 1, 73 \% validity, vs. Experiment 4, $25 \%$ validity) was conducted separately for the ISIs of 50 and $100 \mathrm{~ms}$. The ISIs of 50 and $100 \mathrm{~ms}$ were chosen because significant differences between invalid-inside and invalid-outside were found for these ISIs in at least one the experiments.

\section{ISI of $50 \mathrm{~ms}$}

Accuracy was generally higher for uninformative cues (74.9 \%; Experiment 4) than for informative cues $(63.5 \%$; Experiment 1$), F(1,30)=5.41, p=.027, \eta^{2}=.153$. Additionally, accuracy in both experiments was better on invalid-inside trials $(71.8 \%)$ than on invalid-outside trials $(66.5 \%), F(1,30)=14.27, p=.001, \eta^{2}=.32$. The texture advantage did not differ for informative cues in Experiment 1 $\left(M_{\text {inside }}=66.8 \%\right.$ vs. $\left.M_{\text {outside }}=60.1 \%\right)$, as compared with uninformative cues in Experiment $4\left(M_{\text {inside }}=76.9 \%\right.$ vs. $M_{\text {outside }}=72.9 \%$ ) (interaction of validity and informativeness, $p=.336$ ).

\section{ISI of $100 \mathrm{~ms}$}

The results show the same pattern as for the ISI of $50 \mathrm{~ms}$ : Across both experiments, performance was better for uninformative cues $(75.0 \%$; Experiment 4$)$ than for informative cues $\left(61.7 \%\right.$; Experiment 1), $F(1,30)=7.18, p=.012, \eta^{2}=.193$. In addition, accuracy was higher on invalid-inside trials $(70.2 \%)$ than on invalid-outside trials $(66.6 \%), F(1,28)=5.38, p=.027$, $\eta^{2}=.15$. The texture advantage was of comparable size for informative cues in Experiment $1\left(M_{\text {inside }}=63.5 \%\right.$ vs. $M_{\text {outside }}=$ $60.0 \%)$ and uninformative cues in Experiment $4\left(M_{\text {inside }}=\right.$ $77.0 \%$ vs. $M_{\text {outside }}=73.1 \%$ ); no interaction of validity and informativeness was observed, $p=.907$.

\section{Discussion}

In Experiment 4, the texture advantage was about the same size as in Experiment 1, where the exogenous cue was informative with respect to the subsequent target location. As in Experiment 1, a texture advantage was found for the shortest ISI of $50 \mathrm{~ms}$ but not for longer ISIs of 150 and $200 \mathrm{~ms}$. In contrast to Experiment 1, a texture advantage was also found for an ISI of $100 \mathrm{~ms}$. In Experiment 4, exogenous cues were not informative concerning the subsequent target location; one may conclude that a texture advantage can be observed both in exogenous and endogenous cueing and that an endogenous component is not necessary to elicit this effect.

\section{Experiment 5}

Experiments 1-4 showed that accuracy on invalid trials depended on the texture to which the cued location belonged: When target and cue were part of the same texture, performance was better than when they were in different textures. In these experiments, the texture segregation was identical in cue and search displays; that is, when the visual field in the cue display was segregated into a left and right texture, this segregation into left and right was left unchanged in the search display. Accordingly, we could determine at what point in time the texture advantage disappeared when the texture arrangements were left unchanged. Experiment 5, however, was designed to determine whether and at what point in time the texture advantage would disappear when the segregation changed. To that end, Experiment 5 used different texture arrangements in cue and search displays. Different ISIs between the cue and target allowed for measuring for how long a texture advantage could be observed for the cued texture after textures had been changed.

\section{Method}

The stimuli and procedure were identical to those in Experiment 4, with the following exception: The texture arrangement changed immediately after the cue offset from vertical to horizontal or vice versa. For example, in cue displays, the visual field was segregated in an upper texture 
of horizontal and a lower texture of vertical lines. After cue offset, the visual field changed to a left texture of horizontal and a right texture of vertical lines. Line orientation around the cued location was left unchanged. With this change of texture arrangement, the definition of invalid-inside and invalid-outside changed as well: A target that was presented at a location that was part of the cued texture in the cue display (OLD-invalid-inside; cf. Fig. 3a, middle column) could belong to a different texture during its presentation. Alternatively, a target that was presented at a location outside the cued texture in the cue display could belong to the same texture during its presentation (NEW-invalid-inside; cf. Fig. 3a, right column).

To precisely track the point in time at which the texture advantage may disappear, we used an additional ISI $=0$ condition in which the search display followed the cue display without delay.

Results

\section{Accuracy (cf. Figure 4a and Table 2)}

Valid cues led to most accurate performance $(M=85.7 \%)$, followed by OLD-invalid-inside $(M=79.8 \%)$, NEW-invalidinside $(M=79.5 \%)$, and invalid-diagonal $(M=77.9 \%)$ trials, $F(3,45)=29.24, p<.001, \eta^{2}=.66$. The most accurate performance was reached for an ISI of $0 \mathrm{~ms}(M=86.8 \%)$, followed by ISIs of $50 \mathrm{~ms}(M=85.4 \%), 100 \mathrm{~ms}(M=80.9 \%)$, $150 \mathrm{~ms}(M=76.4 \%)$, and $200 \mathrm{~ms}(M=73.9 \%), F(4,60)=$ $63.35, p<.001, \eta^{2}=.81$. There was no interaction of both factors, $p=.599$. Planned contrasts revealed a significant difference in accuracy between OLD-invalid-inside and NEW-invalid-inside trials for an ISI of $0 \mathrm{~ms}\left(M_{\text {inside }}=\right.$ $87.2 \%$ vs. $\left.M_{\text {outside }}=85.2 \%\right), p=.022, \varepsilon=0.78$, but not for longer ISIs (all $p \mathrm{~s}>.316$ ).

\section{Reaction times (cf. Table 2)}

There was no effect of validity on RT, $F(1.3,19)=0.40$, $p=.586, \eta^{2}=.03$. The shortest RTs were found for an ISI of $200 \mathrm{~ms}(515 \mathrm{~ms})$, followed by ISIs of $150 \mathrm{~ms}(528 \mathrm{~ms})$, $100 \mathrm{~ms}(539 \mathrm{~ms}), 50 \mathrm{~ms}(541 \mathrm{~ms})$, and $0 \mathrm{~ms}(564 \mathrm{~ms}), F(4$, $60)=11.36, p<.001, \eta^{2}=.43$. The interaction of both factors did not reach significance, $p=.101$.

\section{Discussion}

Results showed that only when the ISI was $0 \mathrm{~ms}$ - that is, when the target immediately followed the cue-participants were better on OLD-invalid-inside than on NEW-invalidinside trials. Hence, only immediately after the texture rearrangement could a texture advantage according to the old texture arrangement be observed. With longer ISIs, any texture advantage was lost. At the same time, performance for NEW-invalid-inside trials was not better for any ISI. Thus, although the old texture advantage was lost, no texture advantage according to the novel texture arrangement could evolve once the cue was not visible anymore. Interestingly, the benefit at the cued location (i.e., the traditional cueing effect) persisted throughout all ISIs used, although the texture advantage could not spread to the novel texture.

\section{Experiment 6}

In Experiments 1-4, the textures were defined both by the orientation contrast at the texture border and by the contrast within texture elements - that is, homogeneity within the texture elements. Thus, the texture advantage may have resulted from either of these stimulus characteristics, which may, in turn, have triggered different perceptual processes. Although Experiment 6 was conducted to disentangle these two potential mechanisms by changing texton identity between the cue and search displays, while leaving the orientation contrast at the texture border the same (cf. Fig. 3b). If the texture border alone accounted for the texture advantage in the previous experiments, a similar benefit on invalid-inside trials over invalidoutside trials should be observed. Conversely, if texture element homogeneity (i.e., the identity of all textons within a texture) caused the texture advantage, no benefit in invalidinside trials over invalid-outside trials would be expected.

\section{Method}

The stimuli and procedure were identical to those jn Experiment 4, with one exception: The identity of the line elements was changed from vertical to horizontal and vice versa with cue display offset. For example, when the upper texture consisted of horizontal and the lower texture of vertical lines in the cue display, the upper texture lines would change to vertical and the lower texture lines to horizontal after cue offset. The texture border was left unchanged.

\section{Results}

\section{Accuracy (cf. Figure $4 b$ and Table 2)}

Valid cues led to best performance $(M=84.7 \%)$, while accuracy differed only slightly between the other three conditions: invalid-inside $(M=80.5 \%)$, invalid-outside $(M=$ $80.8 \%)$, and invalid-diagonal $(M=79.6 \%)$ trials, $F(3,45)=$ $12.55, p<.001, \eta^{2}=.46$. The best performance was reached for an ISI of $50 \mathrm{~ms}(M=85.0 \%)$, followed by ISIs of $150 \mathrm{~ms}$ $(M=81.0 \%), 100 \mathrm{~ms}(M=80.9 \%)$, and $200 \mathrm{~ms}(M=78.8 \%)$, $F(3,45)=14.36, p<.001, \eta^{2}=.49$. There was no interaction of both factors, $p=.214$. Planned contrasts revealed no significant 
a Experiment 5

Change of texture arrangement
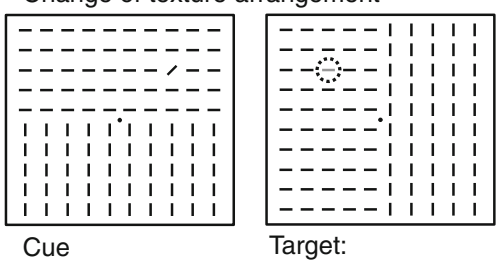

OLD-Invalid-Inside

b Experiment 6

Change of texton identities

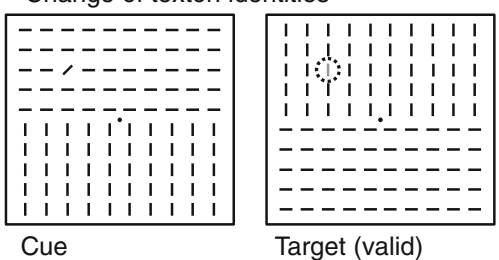

C Experiment 7

Gradual change of texton orientation
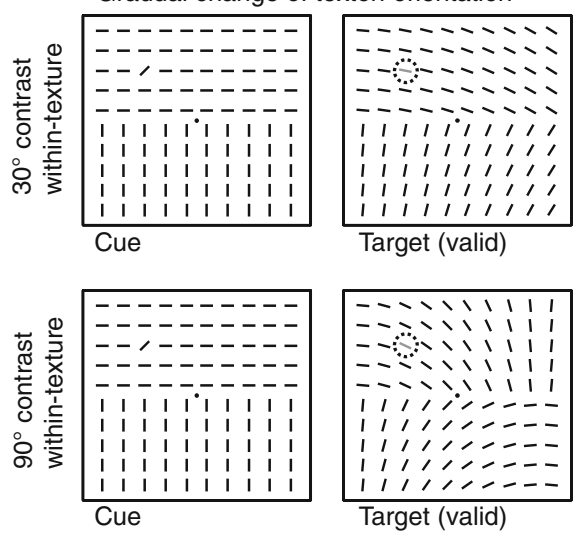

4 Fig. 3 a Illustration of the different texture arrangements in cue and search displays as used in Experiment 5. When the cue display was segregated in an upper texture of horizontal and a lower texture of vertical lines (i.e. horizontal texture border; left panel), the search display (and the standard texture during the interstimulus interval [ISI]) was segregated in a left texture of horizontal and a right texture of vertical lines (i.e., vertical border). Note that this changes the definition of invalid-inside and invalid-outside: A target may be presented at a location that was part of the cued texture in the cue display (OLD-invalid-inside; middle panel) or at a location that would be part of the cued texture if the cue was still present (NEW-invalid-inside; right panel). b Illustration of the textures with texton identities changing between cue and search displays, as used in Experiment 6 . When the cue displays is segregated in an upper texture of horizontal and a lower texture of vertical lines (left panel), the search display (and the standard texture during the ISI) is segregated in an upper texture of vertical and a lower texture of horizontal lines (right panel). Thus, texton identity changes, while the texture border remains unchanged. The dotted line encircles the target locations for illustration purposes and was not visible to the observers. c Illustration of the textures with texton orientation gradually changing, as used in Experiment 7 . The cue display comprised horizontal and vertical lines only. In the search display (and the standard texture during the ISI), the orientation of the line elements gradually changed $30^{\circ}$ (high homogeneity) or $90^{\circ}$ (low homogeneity) from one side of the texture to the other side of the texture. Deviation in orientation (difference in orientation from cue display to orientation in search display) of single line elements increased along the texture border, being small around the cued location and the invalid-outside location and being large around the invalid-inside and invalid-diagonal locations. The increase in deviation was less pronounced in the high-homogeneity condition (upper row) than in the low-homogeneity condition (lower row)

texture segregation (e.g., upper and lower visual fields) in cue and search displays. Thus, the homogeneity within the texture elements seems the critical aspect determining the texture advantage. The texture advantage seems to be bound to the identity of the homogeneously arranged individual textons. The orientation contrast at the border seems less relevant.

\section{Experiment 7}

In Experiment 6, the orientation of each line element changed from horizontal to vertical or vice versa with the cue display offset (i.e., the orientation was swapped for cue display vs. ISI and search display). This resulted in a maximum contrast of $90^{\circ}$ at each location in the display, which may have induced some sort of backward mask. Hence, this mask, rather than the texton identity change as such, could have broken up the texture advantage. In Experiment 7, we wanted to test possible masking effects of the transients by gradually varying the deviation in orientation (cue display search display) within a texture. Invalid-outside trials were privileged over invalid-inside trials by imposing a stronger deviation in orientation at invalid-inside locations. If deviation in orientation served as some sort of mask, this mask should be stronger for invalid-inside, due to the higher deviation in orientation. Let us assume a reliable texture advantage (the advantage for invalid-inside over invalid-outside) the border was left unchanged, generally allowing the same 


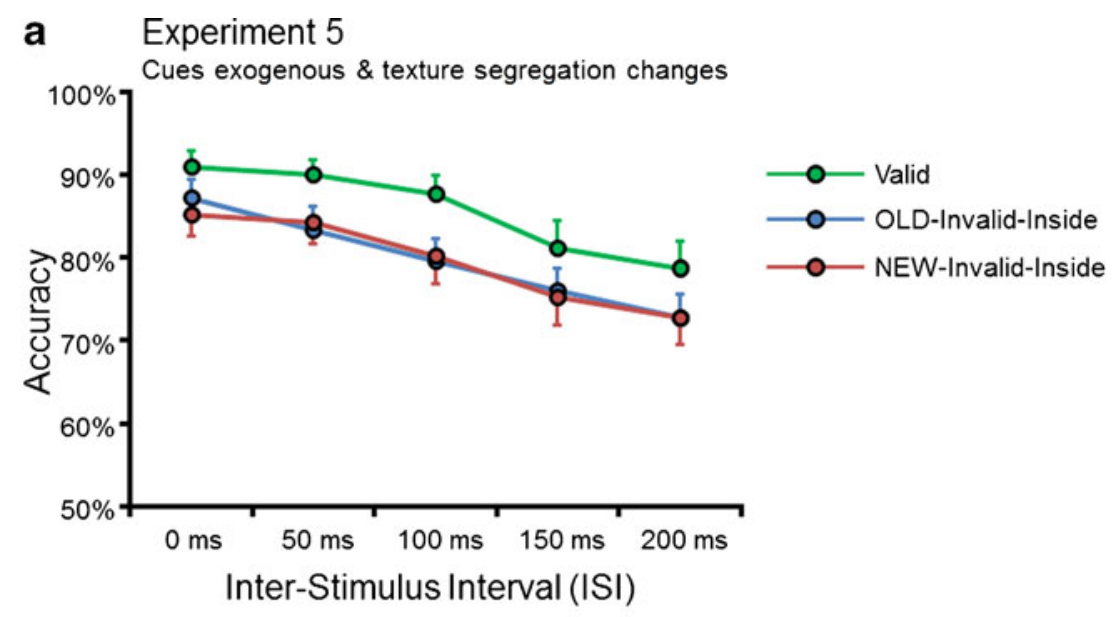

b Experiment 6

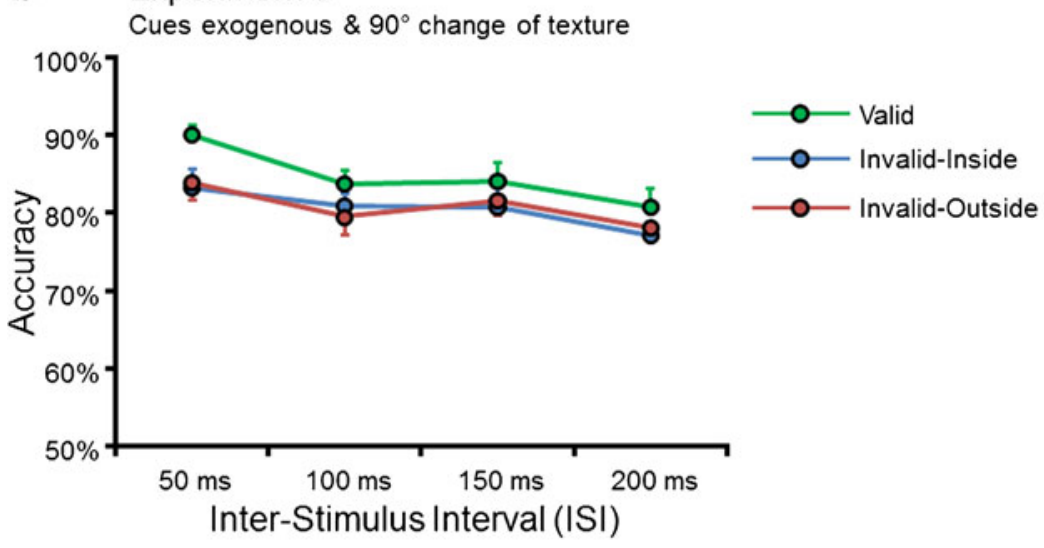

C Experiment 7

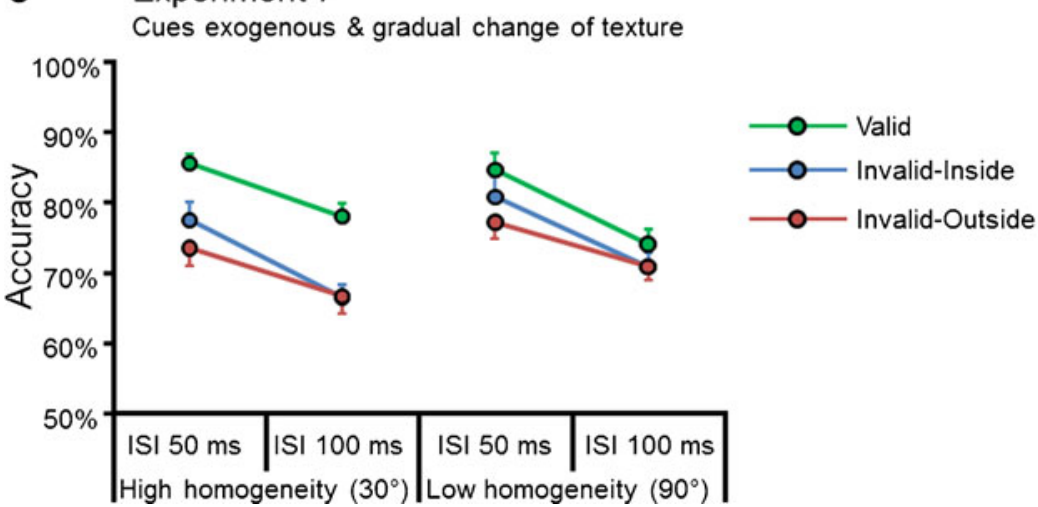

Fig. 4 a Mean accuracy in Experiment 5 as a function of interstimulus interval (ISI) and validity (valid, green lines; invalid-inside, blue lines; invalid-outside, red lines; for invalid-diagonal trials, see Table 2). b Mean accuracy in Experiment 6 as a function of ISI and validity (valid, green lines; OLD-invalid-inside, blue lines; NEW-invalid-inside, red lines; for invalid-diagonal trials, see Table 2). c Mean accuracy in Experiment 7 as a function of ISI, texture homogeneity $\left(30^{\circ}\right.$ vs. $\left.90^{\circ}\right)$, and validity (valid, green lines; invalid-inside, blue lines; invalid-outside, red lines; for invalid-diagonal trials, see Table 2). Error bars indicate the standard errors of the means

\section{Method}

The stimuli and procedure were identical to those in Experiment 6, with one exception: The identity of the line elements was not swapped between the cue and search 
displays from vertical to horizontal or vice versa (i.e., an allencompassing change of $90^{\circ}$ in orientation) but changed gradually within a texture. Deviation of the line orientation from the cue display increased along the border of the textures, with deviation being smallest at the cued location. This deviation manipulation led to varying differences in orientation between the cue display and the search display for the four quadrants of a display (cf. Fig. 3c). For example, in a horizontally divided display, when the cue was presented in the upper left quadrant, the search display lines on the lefthand side (where the cued location was) deviated less from the lines in the cue display than did the lines on the right-hand side (where the invalid-inside location was). To maintain the contrast of $90^{\circ}$ at the texture border, the lower texture changed analogously, leading to a similar gradual deviation in the lower texture from left to right: The search display lines on the left-hand side (where the invalid-outside location was) deviated less from the lines in the cue display than did the lines to the right (where the invalid-diagonal location was). Thus, the deviation manipulation led to the least difference in orientation between the cue display and the search display for valid and invalid-outside trials and to the most difference in orientation for invalid-inside and invalid-diagonal trials. The gradual change from one side of the texture to the other side could be either $30^{\circ}$ (high homogeneity) or $90^{\circ}$ (low homogeneity) (cf. Fig. 3c). If the change in orientation for the search display serves as a backward mask for the cue display, invalidinside locations should suffer more from increased withintexture contrast than should invalid-outside, because the single line element at the invalid-inside location deviated more from the single line element at the same location in the cue display.

\section{Results}

A two-way ANOVA with the factors validity and withintexture contrast (high vs. low) was run for the ISIs of 50 and $100 \mathrm{~ms}$, separately for accuracy and RTs.

\section{ISI 50 ms: Accuracy. (cf. Fig. 4c, Table 2)}

Valid cues led to the best performance $(M=85.2 \%)$, followed by invalid-inside $(M=79.2 \%)$, invalid-diagonal $(M=77.8 \%)$, and invalid-outside $(M=75.4 \%)$ trials, $F(3,45)=10.24$, $p=.001, \eta^{2}=.41$. Performance was slightly better for high within-texture contrasts $(M=80.6 \%)$ than for low withintexture contrasts $(M=78.2 \%), F(1,15)=6.76, p=.020$, $\eta^{2}=.31$. There was no interaction of both factors, $p=.103$. Planned contrasts revealed a significant difference for invalidinside and invalid-outside trials for low within-texture contrast $\left(M_{\text {inside }}=77.6 \%\right.$ vs. $\left.M_{\text {outside }}=73.6 \%\right), p=.002, \varepsilon=0.79$, and for high within-texture contrast $\left(M_{\text {inside }}=80.8 \%\right.$ vs. $M_{\text {outside }}=$ $77.3 \%), p=.018, \varepsilon=0.82$.

\section{ISI $50 \mathrm{~ms}$ : Reaction times (cf. Table 2)}

There were no significant effects (all $p \mathrm{~s} \geq .200$ ).

\section{ISI 100 ms: Accuracy. (cf. Fig. 4c, Table 2)}

Valid cues led to the best performance $(M=76.1 \%)$, while accuracy differed only slightly between the other three conditions: invalid-inside $(M=68.6 \%)$, invalidoutside $(M=68.8 \%)$, and invalid-diagonal $(M=$ $67.6 \%)$ trials, $F(3,45)=5.77, p=.002, \eta^{2}=.28$. Performance was slightly better for high within-texture contrasts $(M=71.5 \%)$ than for low within-texture contrasts $(M=69.0 \%), F(1,15)=10.88, p=.005, \eta^{2}=.42$. While valid cues yielded worse performance for high $(M=$ 74.1) than for low $(M=78.0)$ within-texture contrast, invalid cues yielded better performance for high $(M=$ 70.7) than for low $(M=66.0)$ within-texture contrast [interaction of validity and within-texture contrast, $F(3$, $\left.45)=4.27, p=.010, \eta^{2}=.22\right]$. Planned contrasts revealed no significant difference for invalid-inside and invalidoutside trials for low within-texture or high withintexture contrast (all $p \mathrm{~s} \geq .473$ ).

\section{ISI 100 ms: Reaction times (cf. Table 2)}

There were no significant effects (all $p \mathrm{~s} \geq .113$ ).

\section{Discussion}

In Experiment 7, the textons' identity varied between the cue and search displays, but in contrast to Experiment 6, their deviation in orientation was not always $90^{\circ}$ but depended on the relative position within the texture and was gradually changed. For textons around the cued location and the invalid-outside location, deviation was relatively low, whereas around the invalid-inside location (and the invaliddiagonal location), deviation was relatively high. The results were comparable to those in Experiment 4 (which was similar to Experiment 7, except for the change between the cue and search displays): Invalid-inside trials yielded better performance than did invalid-outside trials for both an ISI of 50 and $100 \mathrm{~ms}$. Valid trials led to best performance. This replication of the texture advantage makes it unlikely that the change in texton identity served as some sort of backward mask that may have broken up the texture advantage in Experiment 6. Since the deviation in orientation from cue display to search display was larger for invalid-inside trials than for invalid-outside trials, presumptive masking should have, if anything, privileged invalid-outside trials, as compared with invalid-inside trials. Quite the contrary was observed: Performance was better on invalid-inside trials than on invalid-outside trials for both high and low homogeneity 
within textures. This texture advantage was almost the same in size for Experiments 4 and 7 (3.95\% for Experiment 4 and $3.75 \%$ for Experiments 7 across ISIs of 50 and $100 \mathrm{~ms}$ ).

This finding is particularly interesting because of its implications for the role of texton identity changes in the texture advantage. In Experiment 6, when the texton identities changed from vertical to horizontal and vice versa, no texture advantage was found. In Experiment 7, when the textons identities also changed, but gradually within a texture, a texture advantage comparable in size to that for unchanged textures (Experiment 4) was found. Most interesting, in the low-homogeneity condition of Experiment 7, the deviation in line orientation from the cue to the search display was about the same as in Experiment 6 for the invalid-inside location. Still, there was a pronounced texture advantage for low-homogeneity trials in Experiment 7 and no texture advantage in Experiment 6. Thus, the difference in performance in Experiments 6 and 7 was likely due to the neighboring elements within the texture. These were gradually decreasing in deviation toward the cued location in Experiment 7 or had exactly the same deviation (i.e., $90^{\circ}$ ) in the entire texture in Experiment 6. Thus, the texture advantage seems not to be bound to the individual identity of the textons. Rather, properties of neighboring textons seem to be crucial for the texture advantage. Results suggest that gradual changes in orientation within a texture do not disrupt processing, whereas abrupt changes in orientation do disrupt processing of the texture. This can lead to differential processing at locations where the actual change of orientation (here, invalid-inside locations) is the same.

\section{Experiment 8}

Experiment 6 suggests that the homogeneity within texture elements contributes more to the texture advantage than does the texture border. A comparison of Experiment 6 and Experiment 7 suggests that not the homogeneity per se but, rather, the properties of neighboring textons within a texture determine how a texture is processed. A gradual change in orientation does not disrupt the texture advantage, whereas a uniform change of the entire texture makes the texture advantage disappear. Experiment 8 investigated the respective proportion of texture homogeneity and texture border in more detail by systematically varying border contrast and texture homogeneity. This texture manipulation goes back to a study by Nothdurft (1992) that employed a texture segregation task. Nothdurft (1992) presented a rectangular patch of simple oriented lines on a background of lines with different orientation and varied the homogeneity of the background (high vs. low homogeneity) independently from the border contrast - that is, the orientation difference of neighboring lines at the border (high vs. low contrast). Although texture homogeneity may rely on various local contrasts within the texture and, thus, be comparable to the contrast at the border, we wanted to disentangle these texture-defining determinants to shed light on differential underlying processes. Thus, we used a similar manipulation as Nothdurft (1992) and varied the homogeneity within texture elements and the orientation contrast at the border independently.

\section{Method}

Similar to Experiment 7, either texture homogeneity could be $30^{\circ}$ (high homogeneity), meaning that line elements always varied within a range of $30^{\circ}$ within a texture (e.g., from $10^{\circ}$ to $40^{\circ}$ ), or texture homogeneity could be $90^{\circ}$ (low homogeneity; e.g., elements varied from $10^{\circ}$ to $100^{\circ}$ ). In contrast to Experiment 6, the texton identity did not change between the cue and search displays. Orientation contrast at the texture border could be $30^{\circ}$ (low contrast), meaning that neighboring lines at each side of the border were $30^{\circ}$ different in orientation (e.g., $0^{\circ}$ vs. $30^{\circ}$ ), or border contrast could be $90^{\circ}$ (as in Experiments 1-6; high contrast; e.g., $0^{\circ}$ vs. $\left.90^{\circ}\right)$. This resulted in $2 \times 2$ possible textures-(1) high homogeneity and high border contrast, (2) high homogeneity and low border contrast, (3) low homogeneity and high border contrast, and (4) low homogeneity and low border contrast (cf. Fig. 5 for an illustration-which were combined with two ISIs ( 50 vs. $100 \mathrm{~ms}$ ), and the four validity conditions from Experiments 1-6. Cues were exogenous and uninformative (cf. Table 1 for all experimental settings). Since there were 2,048 trials, participants had to take part in two sessions of 1,024 trials, each of which was at least $24 \mathrm{~h}$ apart.

\section{Results}

A three-way ANOVA with the factors validity, border contrast (high vs. low), and texture element homogeneity (high vs. low) was run for the ISIs of 50 and $100 \mathrm{~ms}$, separately for accuracy and RTs.

\section{ISI 50 ms: Accuracy (cf. Figure 5 and Table 2)}

Valid cues led to the most accurate performance $(M=92.9 \%)$, followed by invalid-inside $(M=86.5 \%)$, invalid-outside $(M=81.9 \%)$, and invalid-diagonal $(M=81.6 \%)$ trials, $F(1.6,23.6)=11.94, p=.001, \eta^{2}=.44$. No other effects were significant (all $p \mathrm{~s}>.125$ ). Planned contrasts revealed a significant difference in accuracy between invalid-inside and invalidoutside trials when homogeneity was high, both for high border contrast $\left(M_{\text {inside }}=87.9 \%\right.$ vs. $M_{\text {outside }}=81.1 \%$; cf. Fig. 5 , upper left panel), $p=.022, \varepsilon=0.83$, and for low border contrast $\left(M_{\text {inside }}=87.8 \%\right.$ vs. $M_{\text {outside }}=82.2 \%$; cf. Fig. 5 , lower left panel), $p=.012, \varepsilon=0.89$. When homogeneity was low, the 
Experiment 8

Border Contrast \&Texture Homogeneity

Valid

Invalid-Inside

Invalid-Outside

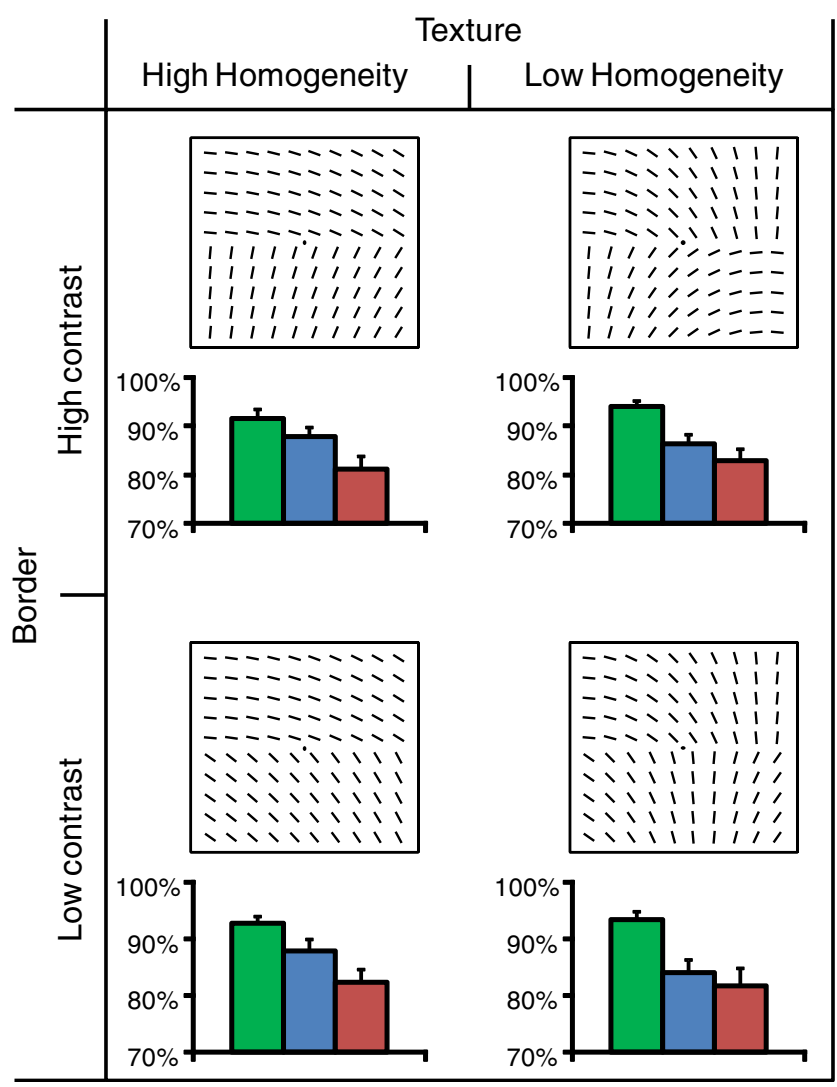

Fig. 5 Mean accuracy in Experiment 8, separately for textures with high (left column) and low homogeneity (right column) and separately for textures with high (upper row) and low border contrasts (lower row). Each of the four panels shows an exemplary texture and the accuracy for each validity condition (valid, green lines; invalid-inside, blue lines; invalid-outside, red lines; for invalid-diagonal trials, see Table 2). The exemplary textures in the four panels all have a "starting angle" of $5^{\circ}$ (upper left texture line) to illustrate how the overall orientation difference between neighboring lines (texture homogeneity) and the local shift in line orientation at the texture border (border contrast) was varied. In the experiment, the "starting angle" was randomly chosen on each trial

difference between invalid-inside and invalid-outside just failed to reach significance for high border contrasts $\left(M_{\text {inside }}=86.4 \%\right.$ vs. $M_{\text {outside }}=82.8 \%$; cf. Fig. 5 , upper right panel), $p=.086$, $\varepsilon=0.51$, while no difference was found for a low border contrasts $\left(M_{\text {inside }}=84.0 \%\right.$ vs. $M_{\text {outside }}=81.6 \%$; cf. Fig. 5 , lower right panel), $p=.197, \varepsilon=0.31$.

\section{ISI 50 ms: Reaction times (cf. Table 2)}

Valid cues led to the shortest RTs (507 ms), while responses differed only slightly between the other three conditions: invalid-inside (542 ms), invalid-outside (546 ms), and invalid- diagonal (544 ms), $F(1.5,22)=10.11, p=.002, \eta^{2}=.40$. No other effects were significant (all $p \mathrm{~s}>.139$ ).

\section{ISI 100 ms: Accuracy (cf. Table 2)}

Valid cues led to the most accurate performance $(M=$ $87.4 \%)$, followed by invalid-inside $(M=80.9 \%)$, invalid-outside $(M=79.5 \%)$, and invalid-diagonal $(M=$ $76.8 \%$ ) trials, $F(1.6,24.4)=10.07, p=.001, \eta^{2}=.40$. No other effects were significant (all $p \mathrm{~s}>.186$ ). Planned contrasts revealed no significant difference in accuracy between invalid-inside and invalid-outside trials for any combination of texture homogeneity and texture border contrast (all $p \mathrm{~s}>$.124).

\section{ISI 100 ms: Reaction times (cf. Table 2)}

There were no significant effects (all $p$ s $>.076$ ).

\section{Discussion}

Experiment 8 systematically varied homogeneity within texture elements and orientation contrast at the texture border. Results showed that textures with high homogeneity (variation within a range of $30^{\circ}$ ) were sufficient to evoke a texture advantage, regardless of the degree of border contrast. In textures with high element homogeneity, targets that were presented in the cued texture (invalid-inside trials) were more often correctly identified than targets in the uncued texture (invalid-outside trials) both for a high border contrast of $90^{\circ}$ (advantage of $6.8 \%$ ) and for a low border contrast of $30^{\circ}(5.6 \%)$. In textures with low element homogeneity (variation of $90^{\circ}$ ), hardly any texture advantage was observed: The effect just failed to reach significance when border contrast was high, and it was absent when border contrast was low. This further supports the notion that texture homogeneity, rather than the orientation contrast at the texture border, caused the texture advantage, although the border may add an additional benefit to invalid-inside, as compared with invalid-outside, trials. Interestingly, although the search display in the high-homogeneity and high-bordercontrast condition in Experiment 8 was identical to the search display in the high-homogeneity condition in Experiment 7, a reliable texture advantage was found only in Experiment 7. The difference between the search displays in both experiments was that the previous cue display was either the same as the search display (Experiment 8) or completely homogeneous (Experiment 7). Apparently, the duration of cue presentation $(100 \mathrm{~ms})$ in which the completely homogeneous texture was shown in Experiment 7 was sufficient to induce a pronounced texture advantage that prevailed over the presentation of the less homogeneous search display. When no completely homogeneous texture 
was shown at all (Experiment 8), the texture advantage could not be fully unfolded.

\section{General discussion}

The present results provide evidence for the impact of textures on the deployment of spatial attention in the visual field. The results indicate that both exogenous and endogenous cues not only enhance processing of subsequently presented targets at cued locations, but also enhance processing of the entire cued texture.

Texture advantage for exogenous and endogenous cues

In eight experiments, participants had to identify the color of a target stimulus. Before search display onset, a cue indicated the likely target position; targets could appear at cued or uncued locations. Both exogenous and endogenous cues resulted in higher performance for subsequent targets when they were presented at the same location as the cue (Experiments 1 and 4-8) or when the endogenous arrow cue was pointing to the target location (Experiments 2 and 3). This shows that the benefit on valid trials that has been observed in traditional cueing experiments (for a review, see Ruz \& Lupiáñez, 2002) can also be obtained by odd elements in otherwise homogeneous textures.

The novel finding of the present experiments is that performance for targets presented at uncued locations systematically depended on the way the visual field was segregated into textures. On invalid trials, performance was still better when the target and cue were presented inside the same texture (invalid-inside) than when they were in different textures (invalid-outside). Note that the spatial distance between the cued location and the target was the same in these conditions, so that the better performance could be attributed only to the position inside or outside the texture. We refer to the accuracy benefit of invalid-inside over invalid-outside as the texture advantage.

We varied the interval between the cue and target (ISI) to track the time course of cueing effects and the texture advantage. When cues were exogenous, the time course of the texture advantage depended on the informativeness of the cue. When the cue was informative, a, texture advantage was found for an ISI of $50 \mathrm{~ms}$, whereas uninformative cues caused a texture advantage for 50 and $100 \mathrm{~ms}$. While it was often argued that exogenous cues can also comprise an endogenous component when being informative (Doallo et al., 2004; Müller \& Rabbitt, 1989; Wright \& Richard, 2000), Experiment 4 could therefore rule out the possibility that an endogenous component is necessary to cause a texture advantage; exogenous control is sufficient. Interestingly, overall performance was worse when cues were informative than when they were uninformative. This might seem odd at first glance, but note that the difference is clearly on invalid rather than valid trials. This is in line with the recent finding that cueing benefits on valid trials might not depend on cue informativeness, whereas costs on invalid trials do depend on cue informativeness: Cueing costs are larger for informative cues than for uninformative cues and can sometimes be found for informative cues only (Doricchi, Macci, Silvetti, \& Macalusa, 2010; Lasaponara, Chica, Lecce, Lupiáñez, \& Doricchi, 2011).

Not only exogenous, but also endogenous cues can trigger a texture advantage as shown in Experiments 2 and 3; however, this effect was observed later than with exogenous cues. For endogenous cues, the time course of the texture advantage depended on the preparation time to deploy attention to a cued location. A texture advantage was found for the shortest ISI of $100 \mathrm{~ms}$ only when preparation time was relatively long (1,000 ms; Experiment 1), but not when it was relatively short (100 ms; Experiment 2). Thus, a longer preparation time that allows participants to optimally prepare for the subsequent target stimulus does not abolish the texture advantage but seems to expand it to an earlier point in time. The longer lasting texture advantage for endogenous than for exogenous cues is in line with the finding that endogenous cues need to be decoded by the organism, leading to delayed attention shifts, as compared with the automatically triggered attention shifts observed with exogenous cues (Müller \& Rabbitt, 1989).

There has been some debate as to how endogenous control relates to object-based attention. Some studies found that only exogenous cues can trigger a spread of attention to entire objects (e.g., Macquistan, 1997; see also Lauwereyns, 1998). However, it was also found that when task demands or instructions encourage a broader attention focus, endogenous cues also can cause object-based attention (Goldsmith \& Yeari, 2003; see also Chen \& Cave, 2008). In contrast to these studies, which used rectangular objects in otherwise empty visual fields, we used fully structured visual fields and textures in the present study. These may, as in Goldsmith and Yeari (2003), have caused a tendency in the observer to generally broaden the attention focus (i.e., independently of task demand or instructions) and to attend entire textures rather than single locations. In any case, the present study provides further evidence that object-based attention may also be obtained under endogenous control.

Contrary to accuracy results, RTs did not differ between invalid-inside and invalid-outside trials in the present experiments. This is not surprising, considering that participants were instructed to respond as correctly as possible, neglecting RTs (which were furthermore not displayed in feedback screens). However, valid trials resulted in shorter RTs, as compared with invalid trials in all experiments. Thus, the traditional cueing effect seems much stronger than the texture advantage and has an impact on RTs even though participants 
did not explicitly try to respond as quickly as possible. Traditional cueing may extend to postperceptual processes such as response selection, while the texture advantage affects mainly perceptual processes.

\section{Attention deployment in a structured visual field}

The present results may shed some new light on the way visual selective attention is shaped by the structure of the visual field. It has previously been demonstrated that attention is rather deployed to entire objects than to specific spatial locations (Duncan, 1984; Egly et al., 1994; O’Craven et al., 1999). For example, when a part of a rectangle is exogenously cued, all rectangle locations benefit from cueing. This suggests that attention tends to spread to an entire object when the object is defined by the Gestalt principles of colinearity and closure (Egly et al., 1994). Similar results were obtained when the visual field was structured by colinearity only (Avrahami, 1999; Marino \& Scholl, 2005) or by multiple objects that differ in similarity (Dodd \& Pratt, 2005).

The present series of experiments extends these findings and shows how attention is deployed on a more fine-grained level. Instead of using full-fledged, rather arbitrarily defined objects that have the shortcoming that is not clear what counts as an object when it comes to the deployment of attention (Ben-Shahar et al., 2007), we structured the visual field with the basic simple feature line orientation, which underlies objects in everyday life. These simple texton stimuli have the advantage that they can be grouped preattentively to be processed as a unit (Julesz, 1986; Nothdurft, 1992, 1993), which allowed separating the effects of segregating the visual field (preattentively into textures) and the deployment of attention (toward cue/target). The texture advantage we found in several experiments indicates that attention is deployed texture-wise. Positions equidistant to a cued location were not provided with the same amount of attention. Instead, targets in the same texture as the cued location were provided with more attention than were targets outside that texture. This is in line with the finding that attention can be divided far better between two locations within the same texture than between two locations within different textures (Ben-Shahar et al., 2007).

The present results show two aspects of the deployment of spatial attention: First, the cued location is provided with more attention than any other location inside or outside its texture, and second, when uncued locations are compared, the texture to which the cued location belongs is provided with more attention than is the other texture. We thus conclude that cueing constituted an attention gradient with highest priority directly at the cued location, followed by less efficiency at locations inside the cued texture, with these receiving yet more priority than locations in the remaining visual field.
For object-based attention, it has been found that after prolonged ISIs, invalid locations that are part of a cued object show impaired performance, as compared with invalid locations that are part of another object, while the cued location still shows enhanced performance (i.e., inhibition of return; Weger, Abrams, Law, \& Pratt, 2008). This was not the case in the present experimental series: Although valid locations showed highest accuracy throughout all ISIs and any texture advantage disappeared after $100 \mathrm{~ms}$ (exogenous cues) or $200 \mathrm{~ms}$ (endogenous cues), performance on invalid-outside trials never exceeded that on invalid-inside trials. This difference in the gradient of attention may be due to the fact that parts of full-fledged objects like rectangles (Weger et al., 2008) are more likely to be subject to inhibition than are parts of a texture (present study). Another reason for not observing inhibition of return in the present experiment may have been the emphasis on accuracy, which usually delays the point in time at which inhibition of return can be observed (Lupiáñez, Milan, Tornay, Madrid, \& Tudela, 1997).

Interestingly, the texture advantage followed a different time course than the traditional cueing effect (Posner, 1980). While traditional cueing was observed for basically all cuetarget intervals, the texture advantage was present for short latencies only. This indicates a different time course of attention deployment. Attention deployment toward cued locations lasts longer than attention deployment toward textures to which the cued location belongs. Results show different time courses of texture-induced gradients of focal attention for exogenous and endogenous cues: Exogenous cues cause a relatively early spread of attention toward the cued texture and then a focus on the cued location for longer ISIs. Endogenous cues cause a strong gradient of attention first, then a broader gradient encompassing the entire texture. Finally, the focus of attention recontracts around the cued location. The results suggest that the size of the attentional gradient is variable, generally shrinks over time, but is also modulated by exogenous versus endogenous control.

\section{The effect of texture grain on the gradient of attention}

To further investigate the texture-induced gradient of attention, we examined whether the grain of the texture affected the way attention was deployed in the visual field. A previous study by Avrahami (1999) has shown that attention deployment in a structured visual field may depend on the grain of the structure. In her experiment, two successive spatially separated stimuli were presented on a grid of long parallel lines that encompassed the entire display. Participants had to respond as quickly as possible whenever they detected the second stimulus. RTs for the second stimulus were shorter when attention had to be shifted parallel to the lines (i.e., a shift "along the grain"; cf. Fig. 6a, upper row), as compared with orthogonal attention shifts (i.e., "against the grain"; cf. 
a

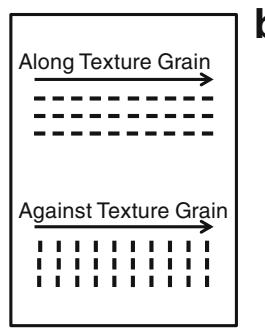

C
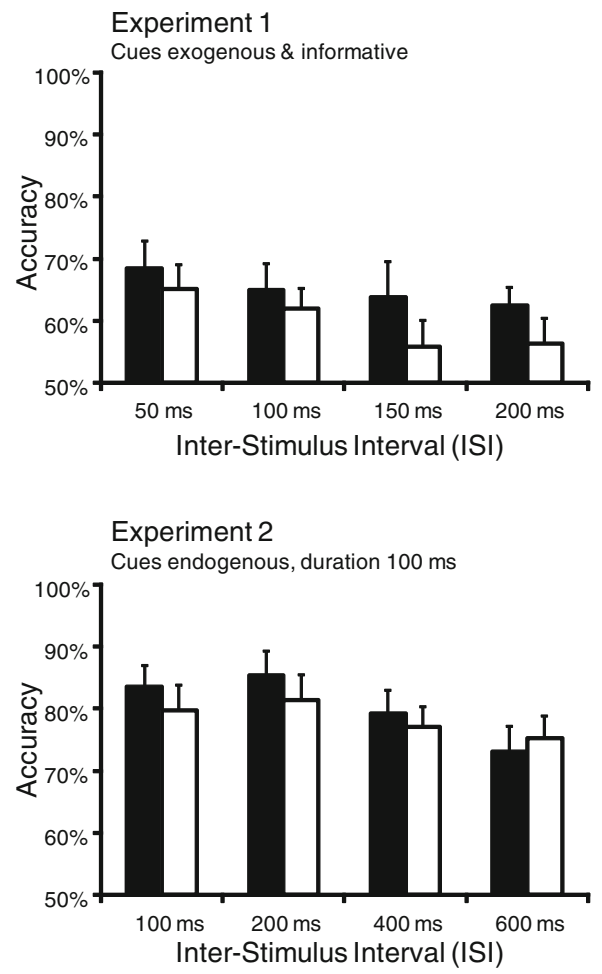

Fig. 6 a Illustrative definition of the texture grain. A direction parallel to the orientation of the lines (upper row) is defined as "Along Texture Grain," whereas a direction orthogonal to the orientation of the lines (lower row) is defined as "Against Texture Grain." b In invalid-inside trials, attention deployment from the cue to the target could be along the grain (left panel) or against the grain (right panel). The dotted line encircles the target locations for illustration purposes and was not visible

Fig. 6a, lower row; Avrahami, 1999), suggesting more efficient attention deployment along the grain than against the grain. Conversely, the grain of a structure does not modulate attention deployment when two to-be-attended stimuli are simultaneously presented (Ben-Shahar et al., 2007). We conducted a post hoc analysis on invalid-inside trials for Experiments 1-4, separately for cues and targets connected along the grain of the texture versus against the grain of the texture (cf. Fig. 6b). Mean accuracy for invalid-inside trials was collapsed across all ISIs and then compared for grain (along vs. against) with a $t$-test for dependent measures for each experiment. In Experiment 1, accuracy was higher for connections along the grain $(M=64.9 \%)$ than for connections against the grain $(M=59.8 \%), p=.036, \varepsilon=0.69$. In Experiment 2, accuracy was only marginally higher for
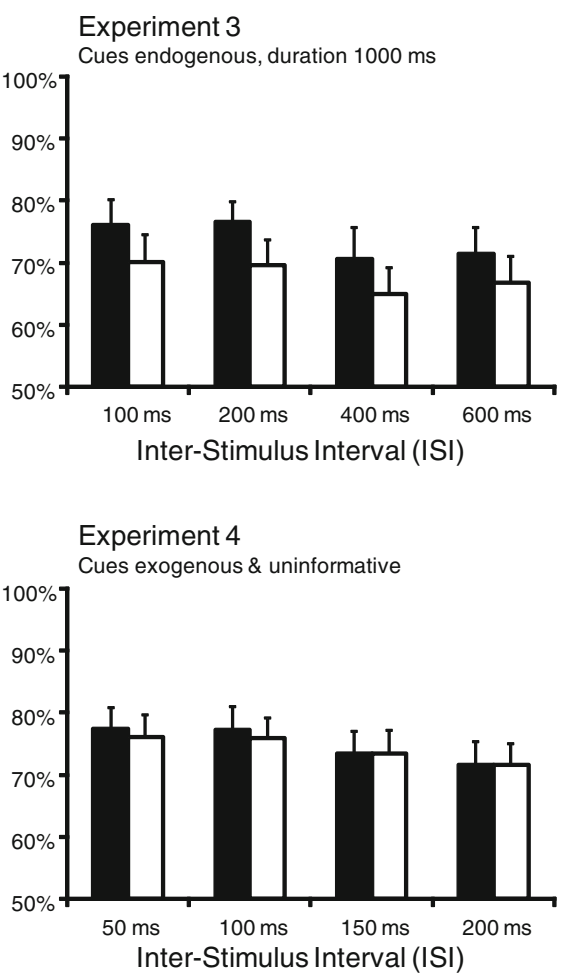

to the participants. $\mathbf{c}$ Mean accuracy for invalid-inside trials in Experiment 1 (upper left panel), Experiment 2 (lower left panel), Experiment 3 (upper right panel), and Experiment 4 (lower right panel). Accuracy is shown as a function of interstimulus interval and attention deployment along the grain (filled bars) versus against the grain (empty bars). Error bars indicate the standard errors of the means

connections along the grain $(M=80.3 \%)$ than for connections against the grain $(M=78.4 \%), p=.088, \varepsilon=0.50$. In Experiment 3, accuracy was higher for connections along the grain $(M=73.7 \%)$ than for connections against the grain $(M=67.9 \%), p<.001, \varepsilon=1.34$. In Experiment 4, accuracy was about equally high for connections along the grain $(M=$ $71.7 \%)$ and for connections against the grain $(M=71.6 \%)$, $p=.449, \varepsilon=0.05$.

These results are well in line with earlier findings (Avrahami, 1999) and show that the texture-induced gradient of attention may also be a function of texture grain. The performance difference between valid and invalid-inside trials was smaller when cue and target locations were connected with the grain, suggesting a more balanced attention deployment within the cued context (i.e., a balanced 
attention gradient). However, for locations connected against the grain, the performance difference between valid and invalid-inside trials was larger, suggesting a more focused attention deployment on the cued location (i.e., a stronger attention gradient). Interestingly, the gradient of attention was modulated by the grain only when cues were informative (Experiments 1, 2, and 3). The differences in invalidinside trials between "against the grain" and "along the grain" may also be interpreted as a figure-ground effect. It has previously been found that textons parallel to the border are more likely to be perceived as the figure and textons orthogonal to the border are more likely to be perceived as the ground (e.g., Jingling \& Zhaoping, 2008). Accordingly, the present results suggest that attention deployment within the figure may be more efficient than attention deployment within the ground.

Representation of texture homogeneity and border contrast on a salience map

Current theories of visual attention often assume that attention deployment is based on a salience map that codes the visual field in a topographical manner; the salience map represents all stimuli in the visual field with a particular activation according to their physical features, such as contrast or similarity (Fecteau \& Munoz, 2006; Itti \& Koch, 2000; Li, 2002; Wolfe, 1994). The activation pattern on the salience map is available at a very early point in visual processing (Itti \& Koch, 2000), may be based on activations in V1 (Li, 2002; Zhaoping, 2003; Zhaoping \& Snowden, 2006), and is used to later prioritize those stimuli in the visual field that will receive more elaborated, attentive processing (e.g., Wolfe, 1994). To account for cueing effects, it has been suggested that targets are represented with a higher activation on valid, as compared with invalid, trials, because of the residual cue activation at the same location (e.g., Fecteau \& Munoz, 2006). Results from the present study support this assumption but also suggest that targets on invalid-inside trials receive more activation than do targets on invalid-outside trials. Apparently, the texture arrangement adds to the cue-induced activations on the salience map and reflects the gradient of attention. While the cued location receives highest activation, the cued texture receives more activation than does the remaining visual field. Thus, on invalid-inside trials, targets benefited from higher residual activation of the cue display, as compared with targets on invalid-inside trials, resulting in the texture advantage.

This activation pattern seems to be disrupted by dynamic changes in the visual field: When the texture arrangement in cue and search displays was different (Experiment 5), no texture advantage was observed, except for the shortest ISI. Apparently, the texture-induced gradient of activation on the salience map rapidly disappears when a novel texture arrangement is shown. Interestingly, the novel texture arrangement does not seem to induce a new gradient of activation on the salience map, since NEW-invalid-inside trials were not found to show better performance than OLDinvalid-inside trials at any ISI. It may be that subsequent texture representations extinguish each other, leaving a single activation peak at the previous cue location merely preserving the traditional cueing effect.

The texture advantage observed in the present experiments may have resulted from orientation border contrast between the two textures (e.g., Zhaoping, 2003) or the homogeneity of texture elements (Nothdurft, 1992). We disentangled these two potential factors by changing texture element (texton) identity between cue and search displays, while leaving the texture border the same. When the deviation in orientation was $90^{\circ}$ (i.e., horizontal and vertical lines were swapped; Experiment 6), results showed that the texture advantage disappeared for all ISIs. When the deviation gradually increased from the cued location to the invalid-inside location (Experiment 7), results showed that this change in identity did not affect the texture advantage. Results from Experiments 6 and 7 suggest that the texture border contrast alone cannot account for the texture advantage. If the texture advantage was due only to the higher activation at the texture border (Zhaoping, 2003), the texture advantage should have been left unchanged in Experiment 6, because the border contrast, inducing higher activation on the salience map (cf. Zhaoping $\&$ Snowden, 2006), was the same on cue and target trials. It is also unlikely that masking effects can account for the lack of texture advantage in Experiment 6: In Experiment 7, where the deviation in orientation (cue display - search display) was higher for invalid-inside trials than for invalid-outside trials, presumptive masking should have privileged, if anything, invalid-outside trials, as compared with invalid-inside trials. However, the disruption of the texture advantage due to a change of texton identity depended on the way the search display varied in orientation. When the deviation in texton orientation gradually varied (Experiment 7 ), texture advantage was stable, while an all-encompassing abrupt change of orientation eliminated the effect. It may be that the presumed spread of higher activation on the salience map from the cued location to all locations that belong to the cued texture was intercepted by the abrupt identity change (Experiment 6), which resulted in lower activation levels at the invalid-inside locations. In Experiment 7, the gradual change in orientation may have allowed a more efficient spread of higher activation from the cued location to all locations that belonged to the cued texture. Results from Experiment 8 showed that such a spread of activation on the salience map within a gradually varying low-homogeneity texture was impaired when the low homogeneity was already present in the cue display. In Experiment 8, orientation contrast at the texture border and homogeneity within texture elements were systematically 
varied. Even for the maximum border contrast of $90^{\circ}$ (also used in Experiments 1-6), only a slight texture advantage was observed when texton homogeneity was low. Conversely, a homogenous texture was sufficient to elicit a texture advantage even for a weak border contrast of $30^{\circ}$. This shows that texture homogeneity had a stronger impact on the texture advantage than did the border. ${ }^{2}$ While border contrast may have some general effect on the distribution of activation on the salience map, the impact of homogeneity on salience map activation is more complex. When the initial cue display is completely homogeneous, a subsequent search display with low homogeneity seems to not interrupt the salience increase of the entire texture as long as changes in orientation are gradual (Experiment 7). When the initial cue display also is less homogeneous, the increase in salience for the entire texture is less pronounced (Experiment 8). It has been argued before that similar stimuli may result in stronger linkage (e.g., Duncan \& Humphreys, 1989; Meinecke \& Donk, 2002; Nothdurft, 1992), which allows grouping into larger perceptual units (Schubö et al., 2004; Schubö, Wykowska, \& Müller, 2007). Dissimilar stimuli, on the other hand, may also result in a high-contrast induced activation on the salience map ( $\mathrm{Li}$, 2002). As a result, activation on the salience map may spread from the cue location to neighboring elements when these are similar enough or gradually changing in orientation, while activation for elements that are too deviant in orientation (e.g., at the border and beyond; the uncued texture) may be suppressed.

Texture advantage as a result of event integration processes

A different approach to account for exogenous cueing effects is the event integration approach (Lupiáñez \& Milliken, 1999; Lupiáñez, Milliken, Solano, Weaver, \& Tipper, 2001). The event integration approach suggests that current perceptual information can be integrated with perceptual information the observer was previously exposed to. This happens in such a way that the spatiotemporal match between successive events (e.g., cue and search displays) determines the efficiency of processing of both events (Funes et al., 2005). According to the event integration approach, cue and target have to have a spatiotemporal match in order to be integrated into one single event. This is the case when the ISI is short enough (temporal match) and the cue is valid (spatial match). In this case, location information from the cue can be used to localize the target, leading to improved target processing (Funes et al., 2005). The spatial match may also be flexibly adjusted to the structure of the entire visual field: Since

\footnotetext{
${ }^{2}$ It should, however, be noted that homogeneity and texture border contrast cannot be completely separated. High texture homogeneity may be similar to a low contrast within a texture, and low texture homogeneity may be similar to a high contrast within a texture.
}

"spatial Stroop," usually reduced by spatial cues, is reduced not only at the cued location, but also for the entire cued object (Luo, Lupiáñez, Funes, \& Fu, 2010), it was suggested that all locations of an object can be integrated into one event. In this regard, the texture advantage found in the present series of experiments could be explained in terms of a spatial match assumption. Spatial match between a cue display and a search display is higher when the cue and target locations change within a texture, as compared with a change between textures. In other words, when the target appears in the same texture as the cue, there may be sufficient spatial match for integration. However, when the target appears in a different texture than the cue, they cannot be perceptually integrated, and two "object representations" have to be initiated, one for the cue and one for the target. For longer ISIs, spatial match might still be evident on invalid-inside trials, while the degree of temporal match would be low on invalid-inside and invalidoutside trials (cf. Luo, Lupiáñez, Funes, \& Fu, 2011). Accordingly, invalid-inside and invalid-outside trials may yield similar accuracy rates at longer latencies. Accordingly, the present data can be well explained in terms of the event integration approach and may be suited to extend it from object-based to texture-based attention.

\section{Conclusions}

There has been quite some evidence that attention deployment is guided by the structure of the visual scene the observer encounters-for example, by colinearity, closure, or similarity of comprised elements (Dodd \& Pratt, 2005; Duncan, 1984; Egly et al., 1994). With a variation of the spatial cueing paradigm (Posner, 1980), the present study investigated in eight experiments how preattentively segregated textures shape the focus of attention induced by spatial cues. Our results showed that not only locations, but also entire textures can benefit from a cue. Whereas highest performance was observed for targets at cued locations (valid trials), a differential performance was shown for invalid trials: for targets presented inside the same texture as the cue, performance was higher, as compared with targets presented outside that texture. This suggests a benefit for stimuli inside the same texture as a previously cued location - that is, a texture advantage. For exogenous cues, this texture advantage was found for informative and uninformative cues. For endogenous cues, the texture advantage was evident no matter whether participants had a short $(100 \mathrm{~ms})$ or a long $(1000 \mathrm{~ms})$ preparation time. Additional experiments revealed that the homogeneity within texture elements may contribute more to this effect than the orientation contrast at the texture border but that, when the orientation changes gradually, a less homogeneous arrangement can still elicit a texture advantage. 
This sheds new light on the way attention is deployed in a structured visual field and helps to explain how the gradient of the attentional focus is shaped by contrast, homogeneity, and gradual changes within a texture.

Author Notes This research was supported by the Deutsche Forschungsgemeinschaft (German Research Foundation; SCHU 1330/6-1) and the Graduate School of Systemic Neurosciences, Munich, and by a $\mathrm{PhD}$ grant to the first author by the Deutsche Studienstiftung. We would like to thank Jeremy Wolfe, Li Zhaoping, and Juan Lupiáñez for excellent comments on a previous version of the manuscript and for helpful suggestions for additional experiments.

\section{References}

Avrahami, J. (1999). Objects of attention, objects of perception. Perception \& Psychophysics, 61, 1604-1612.

Belopolsky, A. V., Zwaan, L., Theeuwes, J., \& Kramer, A. F. (2007). The size of an attentional window modulates attentional capture by color singletons. Psychonomic Bulletin \& Review, 14, 934-938. doi:10.3758/BF03194124

Ben-Shahar, O., Scholl, B. J., \& Zucker, S. W. (2007). Attention, segregation, and textons: Bridging the gap between object-based attention and texton-based segregation. Vision Research, 47, 845860. doi:10.1016/j.visres.2006.10.019

Bergen, J. R., \& Julesz, B. (1983). Parallel versus serial processing in rapid pattern discrimination. Nature, 303, 696-698. doi:10.1038/ 303696a0

Cheal, M. L., \& Lyon, D. R. (1991). Central and peripheral precuing of forced-choice discrimination. Quarterly Journal of Experimental Psychology: Human Experimental Psychology, 43A, 859-880. doi:10.1080/14640749108400960

Chen, Z., \& Cave, K. R. (2008). Object-based attention with endogenous cueing and positional certainty. Perception \& Psychophysics, 70, 1435-1443. doi:10.3758/PP.70.8.1435

Doallo, S., Lorenzo-López, L., Vizoso, C., Rodríguez Holguín, S., Amenedo, E., Bará, S., \& Cadaveira, F. (2004). The time course of the effects of central and peripheral cues on visual processing: An event-related potentials study. Clinical Neurophysiololgy, 115, 199-210. doi:10.1016/S1388-2457(03)00317-1

Dodd, M. D., \& Pratt, J. (2005). Allocating visual attention to grouped objects. European Journal of Cognitive Psychology, 17, 481-497. doi:10.1080/09541440440000122

Doricchi, F., Macci, E., Silvetti, M., \& Macaluso, E. (2010). Neural correlates of the spatial and expectancy components of endogenous and stimulus-driven orienting of attention in the Posner task. Cerebral Cortex, 20(7), 1574-1585. doi:10.1093/cercor/ bhp 215

Downing, C. J., \& Pinker, S. (1985). The spatial structure of visual attention. In M. Posner \& O. Marin (Eds.), Attention and Performance Xl (pp. 171-187). Hillsdale, NJ: Erlbaum.

Duncan, J. (1984). Selective attention and the organization of visual information. Journal of Experimental Psychology. General, 113, 501-517. doi:10.1037/0096-3445.113.4.501

Duncan, J., \& Humphreys, G. W. (1989). Visual search and stimulus similarity. Psychological Review, 96, 433-458. doi:10.1037// 0033-295X.96.3.433

Egly, R., Driver, J., \& Rafal, R. D. (1994). Shifting visual attention between objects and locations: Evidence from normal and parietal lesion subjects. Journal of Experimental Psychology. General, 123, 161-177. doi:10.1037//0096-3445.123.2.161
Eimer, M. (1999). Attending to quadrants and ring-shaped regions: ERP effects of visual attention in different spatial selection tasks. Psychophysiology, 36, 491-503. doi:10.1017/S0048577299980915

Eimer, M. (2000). Event-related brain potentials distinguish processing stages involved in face perception and recognition. Clinical Neurophysiology, 111, 694-705. doi:10.1016/S1388-2457(99) 00285-0

Eriksen, C. W., \& St. James, J. D. (1986). Visual attention within and around the field of focal attention: A zoom lens model. Perception \& Psychophysics, 40, 225-240. doi:10.3758/BF03211502

Fecteau, J. H., \& Munoz, D. P. (2006). Salience, relevance, and firing: A priority map for target selection. Trends in Cognitive Sciences, 10, 382-90. doi:10.1016/j.tics.2006.06.011

Funes, M. J., Lupiáñez, J., \& Milliken, B. (2005). The role of spatial attention and other processes on the magnitude and time course of cueing effects. Cognitive Processing -International Quarterly of Cognitive Science, 6, 98-116. doi:10.1007/s10339-004-0038-7

Ghirardelli, T. G., \& Folk, C. L. (1996). Spatial cuing in a stereoscopic display: Evidence for a "depth-blind" attentional spotlight. Psychonomic Bulletin and Review, 3, 81-86. doi:10.3758/BF03210744

Goldsmith, M., \& Yeari, M. (2003). Modulation of object-based attention by spatial focus under endogenous and exogenous orienting. Journal of Experimental Psychology. Human Perception and Performance, 29, 897-918. doi:10.1037/0096-1523.29.5.897

Itti, L., \& Koch, C. (2000). A saliency-based search mechanism for overt and covert shifts of visual attention. Vision Research, 40, 1489-1506. doi:10.1016/S0042-6989(99)00163-7

Jingling, L., \& Zhaoping, L. (2008). Change detection is easier at texture border bars when they are parallel to the border: Evidence for V1 mechanisms of bottom-up salience. Perception, 37, 197-206. doi:10.1068/p5829

Jonides, J. (1981). Voluntary versus automatic control over the mind's eye's movement. In J. B. Long \& A. D. Baddeley (Eds.), Attention and performance IX (pp. 187-203). Hillsdale, NJ: Erlbaum.

Julesz, B. (1986). Texton gradients: The texton theory revisited. Biological Cybernetics, 54, 245-251

Klein, R. M. (2004). On the control of visual orienting. In M. I. Posner (Ed.), Cognitive neuroscience of attention (pp. 29-44). New York: Guilford Press.

Kravitz, D. J., \& Behrmann, M. (2008). The space of an object: Object attention alters the spatial gradient in the surround. Journal of Experimantal Psychology: Human Perception and Performance, 34, 298-309. doi:10.1037/0096-1523.34.2.298

LaBerge, D. (1983). Spatial extent of attention to letters and words. Journal of Experimental Psychology. Human Perception and Performance, 9, 371-379. doi:10.1037//0096-1523.9.3.371

Lamme, V. A. F. (2000). Neural mechanisms of visual awareness: A linking proposition. Brain and Mind, 1, 385-406. doi:10.1023/ A:1011569019782

Lasaponara, S., Chica, A. B., Lecce, F., Lupianez, J., \& Doricchi, F. (2011). ERP evidence for selective drop in attentional costs in uncertain environments: Challenging a purely premotor account of covert orienting of attention. Neuropsychologia, 49(9), 26482657. doi:10.1016/j.neuropsychologia.2011.05.012

Lauwereyns, J. (1998). Exogenous/endogenous control of space-based/ object-based attention: Four types of visual selection? European Journal of Cognitive Psychology, 10, 41-74. doi:10.1080/713752264

Li, Z. (2002). A saliency map in primary visual cortex. Trends in Cognitive Sciences, 6, 9-16. doi:10.1016/S1364-6613(00)01817-9

Liu, T., Stevens, S. T., \& Carrasco, M. (2007). Comparing the time course and efficacy of spatial and feature-based attention. Vision Research, 47, 108-113. doi:10.1016/j.visres.2006.09.017

Luo, C., Lupiáñez, J., Funes, M. J., \& Fu, X. (2010). Modulation of spatial Stroop by object-based attention but not by space-based attention. Quarterly Journal of Experimental Psychology, 63(3), 516-530. doi:10.1080/17470210903004638 
Luo, C., Lupiáñez, J., Funes, M. J., \& Fu, X. (2011). The modulation of spatial congruency by object-based attention: Analysing the "locus" of the modulation. Quarterly Journal of Experimental Psychology, 64(12), 2455-2469. doi:10.1080/17470218.2011.591935

Lupiáñez, J., Milan, E. G., Tornay, F. J., Madrid, E., \& Tudela, P. (1997). Does IOR occur in discrimination tasks? Yes, it does, but later. Perception \& Psychophysics, 59, 1241-1254.

Lupiáñez, J., \& Milliken, B. (1999). Inhibition of return and the attentional set for integrating vs. differentiating information. Journal of General Psychology, Theme Issue on Visual Attention, Part 2, 126, 392-418.

Lupiáñez, J., Milliken, B., Solano, C., Weaver, B., \& Tipper, S. P. (2001). On the strategic modulation of the time course of facilitation and inhibition of return. Quarterly Journal of Experimental Psychology: Human Experimental Psychology, 54A, 753-773. doi:10.1080/02724980042000453

Macquistan, A. D. (1997). Object-based allocation of visual attention in response to exogenous, but not endogenous, spatial precues. Psychonomic Bulletin \& Review, 4, 512-515. doi:10.3758/BF03214341

Mangun, G. R., \& Hillyard, S. A. (1988). Spatial gradients of visual attention: Behavioral and electrophysiological evidence. Electroencephalography and Clinical Neurophysiology, 70, 417428. doi:10.1016/0013-4694(88)90019-3

Marino, A. C., \& Scholl, B. J. (2005). The role of closure in defining the "objects" of object-based attention. Perception \& Psychophysics, 67, 1140-1149. doi:10.3758/BF03193547

Marzouki, Y., Grainger, J., \& Theeuwes, J. (2007). Exogenous spatial cueing modulates subliminal masked priming. Acta Psychologica, 126, 34-45. doi:10.1016/j.actpsy.2006.11.002

Meinecke, C., \& Donk, M. (2002). Detection perfomance in pop-out task: Nonmonotonic changes with display size and eccentricity. Perception, 31(5), 591-602.

Müller, H. J., \& Findlay, J. M. (1988). The effect of visual attention on peripheral discrimination thresholds in single and multiple element displays. Acta Psychologica, 69(129-1), 55. doi:10.1016/00016918(88)90003-0

Müller, M. M., \& Hübner, R. (2002). Can the spotlight of attention be shaped like a doughnut? Evidence from steady-state visual evoked potentials. Psychological Science, 13, 119-124. doi:10.1111/ 1467-9280.00422

Müller, H. J., \& Rabbitt, P. M. A. (1989). Reflexive and voluntary orienting of visual attention: Time course of activation and resistance to interruption. Journal of Experimental Psychology. Human Perception and Performance, 15, 315-330. doi:10.1037//00961523.15.2.315

Nakayama, K., \& Mackeben, M. (1989). Sustained and transient components of focal visual attention. Vision Research, 29, 1631-1647. doi:10.1016/0042-6989(89)90144-2

Nothdurft, H. C. (1992). Feature analysis and the role of similarity in pre-attentive vision. Perception \& Psychophysics, 52, 355-375.

Nothdurft, H.-C. (1993). The role of features in preattentive vision: Comparison of orientation, motion, and color cues. Vision Research, 33, 1937-1958. doi:10.1016/0042-6989(93)90020-W

O'Craven, K. M., Downing, P. E., \& Kanwisher, N. (1999). fMRI evidence for objects as the units of attentional selection. Nature, 401, 584-587. doi:10.1038/44134
Posner, M. L. (1980). Orienting attention. Quarterly Journal of Experimental Psychology, 32, 3-25. doi:10.1080/00335558008248231

Posner, M. I., Snyder, C. R. R., \& Davidson, B. J. (1980). Attention and the detection of signals. Journal of Experimental Psychology. General, 109, 160-174. doi:10.1037//0096-3445.109.2.160

Ruz, M., \& Lupiáñez, J. (2002). A review of attentional capture: On its automaticity and sensitivity to endogenous control. Psicólogica, 23, 283-309.

Sagi, D., \& Julesz, B. (1987). Short-range limitation on detection of feature differences. Spatial Vision, 2, 39-49. doi:10.1163/ $156856887 \mathrm{X} 00042$

Schubö, A., Schröger, E., \& Meinecke, C. (2004). Texture segmentation and visual search for pop-out targets: An ERP study. Cognitive Brain Research, 21, 317-334. doi:10.1016/j.cogbrainres.2004.06.007

Schubö, A., Wykowska, A., \& Müller, H. J. (2007). Detecting pop-out targets in contexts of varying homogeneity: Investigating homogeneity coding with event-related brain potentials (ERPs). Brain Research, 1138, 136-147.

Shulman, G., Wilson, J., \& Sheehy, J. (1985). Spatial determinants of the distribution of attention. Perception \& Psychophysics, 37, 5966. doi:10.3758/BF03207139

Theeuwes, J. (2004). Top-down search strategies cannot override attentional capture. Psychonomic Bulletin \& Review, 11, 65-70. doi:10. 3758/BF03206462

Theeuwes, J. (2005). Irrelevant Singletons Capture Attention. In L. Itti, G. Rees, \& J. Tsotsos (Eds.), Neurobiology of Attention (pp. 418424). San Diego, CA: Elsevier.

Weger, U. W., Abrams, R. A., Law, M. B., \& Pratt, J. (2008). Attending to objects: Endogenous cues can produce inhibition of return. Visual Cognition, 16, 659-674.

Wolfe, J. M. (1992). "Effortless" texture segmentation and "parallel" visual search are not the same thing. Vision Research, 32, $757-$ 763. doi:10.1016/0042-6989(92)90190-T

Wolfe, J. M. (1994). Guided search 2.0: A revised model of visual search. Psychonomic Bulletin \& Review, 1, 202-238. doi:10.3758/ BF03200774

Wright, R. D., \& Richard, C. M. (2000). Location cue validity affects inhibition of return of visual processing. Vision Research, 40, 2351-2358. doi:10.1016/S0042-6989(00)00085-7

Wright, R. D., \& Ward, L. M. (2008). Orienting of Attention. Oxford/ New York: Oxford University Press.

Yantis, S., \& Jonides, J. (1990). Abrupt visual onsets and selective attention: Voluntary versus automatic allocation. Journal of Experimental Psychology. Human Perception and Performance, 16, 131-134. doi:10.1037//0096-1523.16.1.121

Yeshurun, Y., Montagna, B., \& Carrasco, M. (2008). On the flexibility of sustained attention and its effects on a texture segmentation task. Vision Research, 48, 80-95. doi:10.1016/j.visres.2007.10.015

Zhaoping L (2003) V1 mechanisms and some figure-ground and border effects. Journal of Physiology 503-515. doi:10.1016/j.jphysparis. 2004.01.008

Zhaoping, L., \& Snowden, R. J. (2006). A theory of a saliency map in primary visual cortex (V1) tested by psychophysics of colororientation interference in texture segmentation. Visual Cognition, 14, 911-933. doi:10.1080/13506280500196035 